\title{
No evidence for significant age spreads in young massive LMC clusters ${ }^{\star}$
}

\author{
F. Niederhofer ${ }^{1,2}$, M. Hilker ${ }^{1}$, N. Bastian ${ }^{3}$, and E. Silva-Villa ${ }^{4,5}$ \\ ${ }^{1}$ European Southern Observatory, Karl-Schwarzschild-Straße 2, 85748 Garching bei München, Germany \\ e-mail: fniederh@eso.org \\ 2 Universitäts-Sternwarte München, Scheinerstraße 1, 81679 München, Germany \\ 3 Astrophysics Research Institute, Liverpool John Moores University, 146 Brownlow Hill, Liverpool L3 5RF, UK \\ ${ }^{4}$ Centre de Recherche en Astrophysique du Québec (CRAQ), Université Laval, 1045 Avenue de la Médecine, G1V 0A6 Québec, \\ Canada \\ ${ }^{5}$ FACom-Instituto de Física-FCEN, Universidad de Antioquia, Calle 70 No. 52-21, Medellín, Colombia
}

Received 23 June 2014 / Accepted 20 December 2014

\begin{abstract}
Recent discoveries have put the picture of stellar clusters being simple stellar populations into question. In particular, the colormagnitude diagrams of intermediate age (1-2 Gyr) massive clusters in the Large Magellanic Cloud (LMC) show features that could be interpreted as age spreads of 100-500 Myr. If multiple generations of stars are present in these clusters then, as a consequence, young $(<1 \mathrm{Gyr})$ clusters with similar properties should have age spreads of the same order. In this paper we use archival Hubble Space Telescope (HST) data of eight young massive LMC clusters (NGC 1831, NGC 1847, NGC 1850, NGC 2004, NGC 2100, NGC 2136, NGC 2157 and NGC 2249) to test this hypothesis. We analyzed the color-magnitude diagrams of these clusters and fitted their star formation history to derive upper limits of potential age spreads. We find that none of the clusters analyzed in this work shows evidence for an extended star formation history that would be consistent with the age spreads proposed for intermediate age LMC clusters. Tests with artificial single age clusters show that the fitted age dispersion of the youngest clusters is consistent with spreads that are purely induced by photometric errors. As an additional result we determined a new age of NGC 1850 of $\sim 100 \mathrm{Myr}$, significantly higher than the commonly used value of about $30 \mathrm{Myr}$, although consistent with early HST estimates.
\end{abstract}

Key words. galaxies: star clusters: general - Magellanic Clouds - Hertzsprung-Russell and C-M diagrams - stars: evolution

\section{Introduction}

Globular clusters (GCs) have long been thought to be simple stellar populations (SSP), as their stars formed out of the same molecular cloud at approximately the same time. Therefore all stars in a GC should have (within a small range) the same age and the same chemical composition. However, recent discoveries have put this simple picture into question. Features like extended main sequence turn-offs (MSTOs), double main sequences (MS) and multiple subgiant and giant branches in the color-magnitude diagrams (CMDs) of GCs as well as abundance variations of light elements (e.g. C, N, O, Na, Al) have been found (e.g. Gratton et al. 2012; Piotto et al. 2012). There are several attempts to explain the observed anomalies in GCs. The most common model implies the presence of multiple generations of stars inside GCs. In this model, the second generation of stars is thought to be formed out of enriched material from the first stellar generation. Proposed sources of the ejecta are AGB stars (D'Ercole et al. 2008), fast rotating stars (Decressin et al. 2009) and interacting binaries (de Mink et al. 2009). A large drawback of this model, however, is that in order to produce the observed amount of second generation stars the cluster

\footnotetext{
* Based on observations made with the NASA/ESA Hubble Space Telescope, and obtained from the Hubble Legacy Archive, which is a collaboration between the Space Telescope Science Institute (STScI/NASA), the Space Telescope European Coordinating Facility (ST-ECF/ESA) and the Canadian Astronomy Data Centre (CADC/NRC/CSA)
}

must have been 10-100 times more massive in the past than observed today (Conroy 2012). An alternative scenario has been proposed by Bastian et al. (2013a) where chemically enriched material from rotating stars and massive interacting binaries falls on to the accretion disks of low-mass pre-MS stars of the same generation and is finally accreted on the still forming stars. In this scenario all stars are from the same generation without significant age spreads among them.

Besides the anomalous features observed in old GCs which point towards a more complex scenario than a single stellar population, intermediate age (1-2 Gyr) clusters in the LMC show extended or double MSTOs (e.g. Mackey \& Broby Nielsen 2007; Mackey et al. 2008; Milone et al. 2009; Goudfrooij et al. 2009, 2011a,b). This might be a consequence of an extended period of star formation (100-500 Myr). Other theories relate the extended MSTOs to interacting binaries (e.g. Yang et al. 2011) or stellar rotation (e.g. Bastian \& de Mink 2009; Yang et al. 2013; $\mathrm{Li}$ et al. 2014). The centrifugal force in rotating stars decreases the effective gravity which causes a lower effective temperature and luminosity (e.g. Meynet \& Maeder 1997). However, Platais et al. (2012) analyzed the CMD of the galactic open cluster Trumpler 20 and did not find evidence that rotation affects the CMD. Possibly, extended or multiple MSTOs in intermediate age clusters are associated with the presence of multiple stellar populations observed in old GCs (Conroy \& Spergel 2011).

However, recent studies suggest that intermediate age LMC clusters, in contrast to galactic GCs, do not have spreads in chemical abundances. Mucciarelli et al. (2008) analyzed the 
extended MSTO clusters NGC 1651, NGC 1783 and NGC 2173 , and also the older cluster NGC 1978 and did not find chemical anomalies. Furthermore, NGC 1866 (Mucciarelli et al. 2011), NGC 1806 (Mucciarelli et al. 2014) and NGC 1846 (Mackey et al., in prep.) also do not have abundance spreads. This calls into question the idea that the extended MSTO feature is due to age spreads, as such scenarios would naturally predict abundance spreads through self-pollution. Thus, an understanding of the properties of intermediate age clusters can give valuable insight in the evolution of GCs.

If it is true that intermediate age clusters host multiple stellar generations then young $(<1 \mathrm{Gyr}$ ) clusters with similar properties should display signs of age spreads inside the cluster as well. There are several studies that search for age spreads or ongoing star formation in young massive clusters (YMCs). Cabrera-Ziri et al. (2014) analyzed the spectrum of a YMC in the merger galaxy NGC 34 and concluded that the star formation history (SFH) is consistent with a single stellar population. Bastian et al. (2013b) presented a catalog containing more than 100 galactic and extragalactic young ( $<100 \mathrm{Myr})$ massive clusters and did not find any evidence of ongoing star formation in their sample.

Bastian \& Silva-Villa (2013) started a project to constrain possible age spreads in young massive LMC clusters. They analyzed the CMDs of the two young clusters NGC 1856 (281 Myr) and NGC 1866 (177 Myr) and fitted SFHs to the clusters' CMDs. They found no age spreads in these two clusters and concluded that both are consistent with a single burst of star formation that lasted less than $35 \mathrm{Myr}$. In this work, we continue the study by Bastian \& Silva-Villa (2013) by searching for potential age spreads in eight more YMCs in the LMC.

We studied the CMDs of the clusters and fitted theoretical isochrones to them to determine their age, metallicity, extinction and distance modulus (DM). With these parameters we created model Hess diagrams of different ages and compared them with the observations to put initial constraints on any age spread that might be present within the clusters. In order to provide more quantitative results, we also fitted the SFH of each cluster using the code FITSFH (Silva-Villa \& Larsen 2010; Larsen et al. 2011). This code creates a theoretical Hess diagram taking into account the photometric errors and some assumptions (e.g. metallicity). It searches for the best match between the data and a theoretical model which is a linear combination of Hess diagrams constructed from different isochrones. We do not take into account binaries and differential reddening (except for NGC 2100 which has a considerable variation of extinction across the cluster) in the fitting of the SFH. Their effects on the CMDs will only increase any potential age spread that might be present in the clusters. Additionally, the archival photometric data tables that we use for this work contain only the standard deviation of the data reduction as photometric errors. Figure 1 shows, as an example, the errors in $B-V$ color of NGC 2136 as a function of the $V$-band magnitude. The filled squares (red) indicate the width of the MS, given by the standard deviation. The width of the MS is consistent with the overall trend of the errors, especially in the region $17 \leq V$ [mag] $\leq 19$ that is not sensitive to age. The real scatter of the errors around the global relation might be larger as the photometric standard deviations underestimate the real errors. However, underestimating the photometric errors will also lead to larger estimated age spreads. Therefore, all age spreads presented in this work are upper limits of real ones in the clusters. The aim of this work is not to quantify the exact extent of the SFH, but rather to test if significant (tens to hundreds of Myr) age spreads are present within the clusters. For all models and fittings in this paper we used the Parsec 1.1

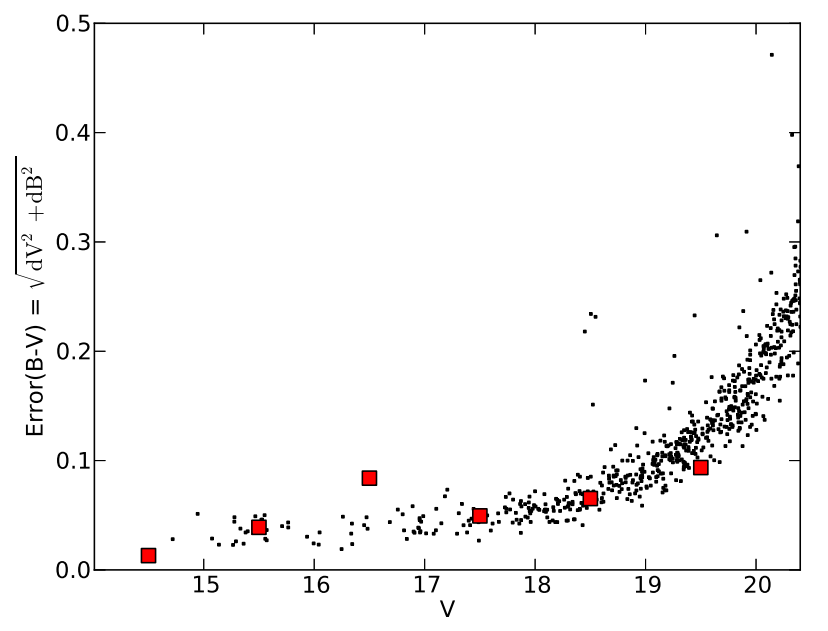

Fig. 1. Color errors $\left(\sqrt{\mathrm{d} B^{2}+\mathrm{d} V^{2}}\right)$ of NGC 2136 as a function of the $V$-band magnitude. The filled (red) squares indicate the dispersion width of the main sequence.

isochrone set of the Padova isochrones (Bressan et al. 2012) and we assumed a Salpeter (1955) IMF.

The structure of the paper is the following: Sect. 2 describes the used data set and the further processing of the data. We perform tests with artificial clusters in Sect. 3. The results of the fitting of the SFH are given in Sect. 4. We discuss the results and draw final conclusions in Sect. 5.

\section{Observations and data processing}

For our analysis we made use of data taken with the Wide Field and Planetary Camera 2 (WFPC2) on board of the Hubble Space Telescope (HST). The data set is presented in detail in Brocato et al. (2001) and Fischer et al. (1998). NGC 1850, however, is not part of these studies, we retrieved the data tables of this cluster from the HST Legacy Archive (HST Proposal 5475, PI: M. Shara). NGC 2157 is presented in Fischer et al. (1998) whereas the Brocato et al. (2001) data set covers the other six clusters. The images of the clusters were taken in the $F 450 \mathrm{~W}$ and $F 555 W$ filters (Brocato et al. 2001 data set and NGC 1850) and in the $F 555 W$ and $F 814 W$ filters (Fischer et al. 1998). All the magnitudes in the HST filter system were transformed to standard Johnson $B V I$ magnitudes. We use the fully reduced data tables which consist of the stars' $x$ and $y$ pixel coordinates on the detector system, the magnitudes and their standard deviations in two filters each.

The HLA catalog of NGC 1850 does not include photometric errors. So we created artificial errors that were modeled from the Brocato et al. (2001) data set. We took these clusters from the data set that have the same exposure time (40 s) as NGC 1850 as a reference and we simulated the errors such that they follow the same exponential increase with fainter magnitudes as the errors from the reference clusters. Figure 2 shows the artificial errors in $B-V$ color as a function of the $V$-band magnitude. The filled (red) squares indicate the width of the MS (standard deviation) of NGC 1850. It is comparable with the mean error in the interval $17 \leq V[\mathrm{mag}] \leq 19$ where the MS is not affected by age effects. We are aware that the scatter of the modeled errors could also be underestimated as the errors were modeled from the standard deviation of the photometric reduction of other clusters (see previous section).

Additionally to the photometry of NGC 2157, Fischer et al. (1998) provide data of a background field which is located $26^{\prime \prime}$ east and $110^{\prime \prime}$ north of the cluster for subtraction of 
Table 1. Parameters of the LMC clusters.

\begin{tabular}{|c|c|c|c|c|c|c|c|c|c|c|}
\hline \multirow[t]{2}{*}{ Cluster } & \multicolumn{2}{|c|}{ Age (Myr) } & \multirow[t]{2}{*}{$\log$ Mass $/ M_{\odot}$} & \multicolumn{2}{|c|}{$Z\left(Z_{\odot}=0.0152\right)$} & \multirow[t]{2}{*}{$R_{\text {core }}(\mathrm{pc})$} & \multirow[t]{2}{*}{$V_{\mathrm{esc}}\left(\mathrm{km} \mathrm{s}^{-1}\right)$} & \multirow{2}{*}{$\begin{array}{c}V_{\text {esc }}\left(\mathrm{km} \mathrm{s}^{-1}\right) \\
\text { at } 10 \mathrm{Myr}\end{array}$} & \multicolumn{2}{|c|}{$E(B-V)$} \\
\hline & lit. & this work & & lit. & this work & & & & lit. & this work \\
\hline NGC 1831 & $700^{a}$ & 926 & $4.59^{b}$ & $0.016^{a}$ & 0.016 & $4.24^{c} / 4.13^{b}$ & $6.6^{b}$ & 9.3 & $0.01^{a}$ & 0.0 \\
\hline NGC 1847 & $26^{d}$ & 57 & $4.44^{b}$ & $0.006^{e}$ & 0.006 & $3.35^{c} / 1.73^{b}$ & $4.2^{b}$ & 4.5 & $0.1^{f}$ & 0.16 \\
\hline NGC 1850 & $30^{g}$ & 93 & $4.86^{b} / 5.15$ & $0.008^{h}$ & 0.006 & $--/ 2.69^{b}$ & $8.77^{b} / 12.3$ & 15.5 & $0.17^{h}$ & 0.1 \\
\hline NGC 2004 & $20^{i}$ & 20 & $4.36^{b}$ & $0.004^{j}$ & 0.004 & $2.18^{c} / 1.41^{b}$ & $7.0^{b}$ & 7.5 & $0.08^{k}$ & 0.23 \\
\hline NGC 2100 & $16^{i}$ & 21 & $4.36^{b}$ & $0.007^{j}$ & 0.007 & $3.03^{c} / 0.99^{b}$ & $7.9^{b}$ & 8.5 & $0.24^{k}$ & 0.17 \\
\hline NGC 2136 & $100^{l}$ & 124 & $4.30^{b}$ & $0.004^{l}$ & 0.005 & $2.91^{c} / 1.59^{b}$ & $7.4^{b}$ & 9.3 & $0.1^{l}$ & 0.13 \\
\hline NGC 2157 & $100^{m}$ & 99 & $4.31^{b}$ & $0.008^{m}$ & 0.008 & $--/ 2.00^{b}$ & $6.2^{b}$ & 7.8 & $0.1^{m}$ & 0.1 \\
\hline NGC 2249 & $1000^{n}$ & 1110 & $4.48^{n}$ & $0.007^{a}$ & 0.008 & $2.79^{c} / 1.75^{b}$ & $9.4^{n}$ & 12.1 & $0.01^{a}$ & 0.02 \\
\hline NGC 1856 & \multicolumn{2}{|c|}{$281^{\circ}$} & $4.88^{b}$ & \multicolumn{2}{|c|}{$0.008^{\circ}$} & $--/ 1.75^{b}$ & $11.0^{b}$ & 14.7 & \multicolumn{2}{|c|}{$0.26^{\circ}$} \\
\hline NGC 1866 & \multicolumn{2}{|c|}{$177^{\circ}$} & $4.91^{b}$ & \multicolumn{2}{|c|}{$0.008^{\circ}$} & $--/ 2.79^{b}$ & $9.5^{b}$ & 12.5 & \multicolumn{2}{|c|}{$0.05^{\circ}$} \\
\hline
\end{tabular}

Notes. In the columns that give the age, the metallicity $Z$ and the extinction $E(B-V)$, the first value is from the literature and the second one is determined in this work. Additionally, we also found new values for the mass and the escape velocity of NGC 1850 (second values in the respective columns). The core radius of the clusters can be determined in two ways: using the surface number density (first value) or the surface brightness density (second value). The last two clusters (NGC 1856 and NGC 1866) are not studied in this work. They were already analyzed by Bastian \& Silva-Villa (2013).

References. ${ }^{(a)}$ Kerber et al. (2007); ${ }^{(b)}$ McLaughlin \& van der Marel (2005) assuming King (1966) profiles; ${ }^{(c)}$ Brocato et al. (2001); ${ }^{(d)}$ Elson \& Fall (1988); ${ }^{(e)}$ Mackey \& Gilmore (2003); ${ }^{(f)}$ Nelson \& Hodge (1983); ${ }^{(g)}$ Baumgardt et al. (2013); ${ }^{(h)}$ Fischer et al. (1993); ${ }^{(i)}$ Elson (1991); ${ }^{(j)}$ Jasniewicz \& Thevenin (1994); ${ }^{(k)}$ Keller et al. (2000); ${ }^{(l)}$ Dirsch et al. (2000); ${ }^{(m)}$ Fischer et al. (1998); ${ }^{(n)}$ Correnti et al. (2014); ${ }^{(o)}$ Bastian \& Silva-Villa (2013).

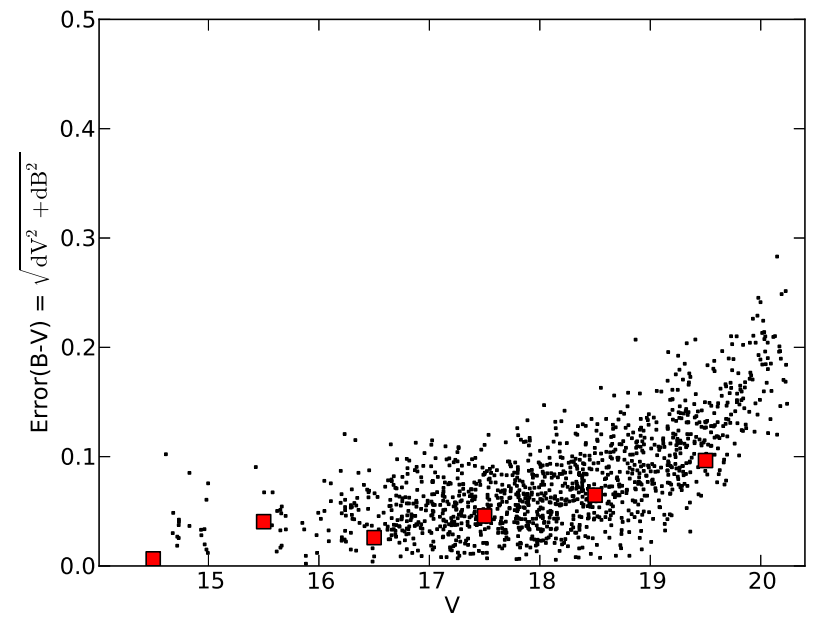

Fig. 2. Modeled color errors $\left(\sqrt{\mathrm{d} B^{2}+\mathrm{d} V^{2}}\right)$ of NGC 1850 as a function of the $V$-band magnitude. The filled (red) squares indicate the observed dispersion width of the main sequence.

field stars. For our analysis we restrict ourselves to the inner regions of the clusters.

As the clusters in the Brocato et al. (2001) data set and NGC 1850 are not centered on any of the four WFPC2 detector chips we had to determine their cluster centers in the $x, y$ CCD coordinate system. We did this by first creating artificial blurred images of the clusters by convolving the flux weighted spatial positions of the stars in each cluster with a Gaussian. We chose Gaussians with a $\sigma$ ranging between 40 and 60 pixels (changing from cluster to cluster) in order to get a smooth flux distribution in the center of each cluster. Afterwards we fitted elliptical isophotes to the created images using the IRAF ${ }^{1}$ task ellipse and used the center of the innermost isophotes as the cluster center. Only those stars which are 1 IRAF is distributed by the National Optical Astronomy
Observatories, which is operated by the Association of Universities for
Research in Astronomy, Inc., under cooperative agreement with the national Science Foundation. located inside two times the core radius $R_{\text {core }}$ (radius at which the density is half the central density) given by Brocato et al. (2001) are used for further analysis. $R_{\text {core }}$ is between 2.0 and $\sim 4 \mathrm{pc}$ (see Table 1), at an assumed distance of $50 \mathrm{kpc}$ (de Grijs et al. 2014). NGC 2157, however, is approximately centered on the PC chip of the WFPC2. The stellar density is highest on this chip with a steep fall off towards the other three chips. For this cluster we use just the stars located on the PC chip which has $800 \times 800$ pixels with a pixel scale of 0 ! $^{\prime} 046$ per pixel. Assuming a distance of $50 \mathrm{kpc}$ to the LMC the PC chip covers an area of $8.9 \times 8.9 \mathrm{pc}$. This area is comparable with the area given by the projected halflight radius of NGC 2157 (5.4 pc; McLaughlin \& van der Marel 2005 assuming King 1966 models).

For our purpose we need to further analyze the data sets. The first step is the subtraction of the field star contamination which is a combination of Galactic foreground stars and LMC field stars. For all the clusters, except for NGC 2157, we do not have extra field exposures so we have to use the cluster images themselves for the field star subtraction. To minimize the contribution of cluster stars we constructed areas that are as far away from the center of the cluster as possible. We chose annuli centered on the respective cluster that intersect the corners of the chips such that the intersection area of the annulus and the chip is approximately the same as the area where we want to subtract the contaminating stellar population (see Fig. 3 as an example). After defining the areas we created CMDs for the stars in both regions and subtracted for every star in the field CMD the star in the corresponding cluster CMD that has the closest geometrical distance in color-magnitude space. However, we have to bear in mind that also in this outer regions a certain fraction of cluster stars is still present that is subtracted as field stars. This becomes clear if we compare the field of view of the WFPC2 detector with the tidal radii of the clusters. The tidal radii are always larger than $30 \mathrm{pc}$ (McLaughlin \& van der Marel 2005; assuming a King 1966 profile) whereas the field of view of the WFPC2 is $\sim 36 \mathrm{pc}$ in diameter (assuming a distance of $50 \mathrm{kpc}$ to the LMC). However, an over-subtraction is not a serious issue as it affects mostly the well populated regions in the CMDs and keeps the overall structure unchanged. 


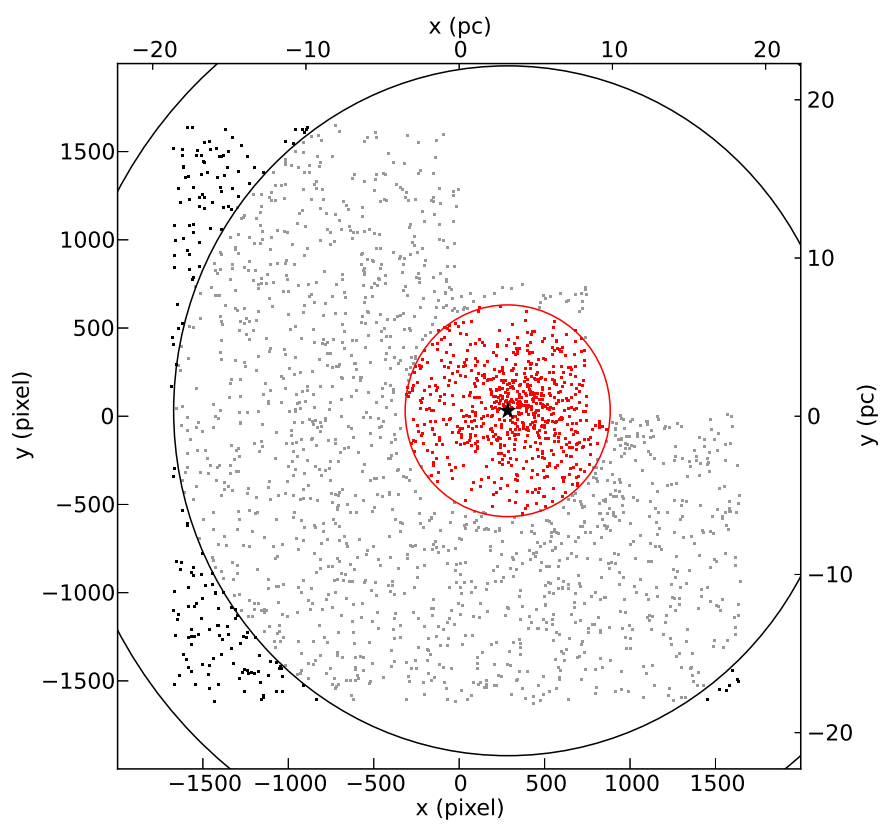

Fig. 3. NGC 1847; Positions of the stars on the WFPC2 chip. The inner circle (red) corresponds to two times the core radius of the cluster. The intersection of the outer two circles forming an annulus with the corners of the WFPC2 field of view is the area which is used for field star removal. The asterisk symbol marks the center of the cluster as determined by us.

As already mentioned we are provided with an image of field stars near NGC 2157. Therefore, in this case, we made CMDs of the stars in the field and the cluster region of every chip and subtracted the field stellar population of every chip separately the same way as we did for the previous clusters. Figure 4 shows the CMDs of all clusters. The black dots indicate all stars that were used in our analysis and the triangles (cyan) are the subtracted "field" stars.

Brocato et al. (2001) and Fischer et al. (1998) performed artificial star tests to infer the completeness curves of their clusters. We adopt limiting $V$-band magnitudes that are at or brighter than the $90 \%$ completeness limit (cf. their Table 3). Fischer et al. (1998) determined for NGC 2157 a 90\% completeness limit in the $I$ band of $\sim 20.5$ mag (cf. their Fig. 3). For NGC 1850, however, we do not have any measure of the completeness. To be on the safe side, we choose a limit of $18.5 \mathrm{mag}$ in the $V$ band for the further analysis of this cluster. This limit is more than $1 \mathrm{mag}$ brighter than the completeness limits of the cluster in the Brocato et al. (2001) data set with comparable exposure times.

\section{Tests with artificial star clusters}

Before we fit the SFH of our sample of LMC clusters we made tests with artificial coeval star clusters to assess the magnitude of apparent age spreads that are purely induced by photometric errors. For each cluster in our sample we modeled a corresponding artificial cluster with the same metallicity and age that we found for the real cluster. We assigned every cluster a random number of stars that is drawn from a Gaussian distribution that peaks at the number of stars present in the data table of the respective observed cluster. The masses of the cluster stars were drawn stochastically from a stellar initial mass function (IMF) with an index $\alpha$ of -2.35 (Salpeter 1955). The lower limits of the stellar masses were chosen such that the CMDs of the artificial clusters cover the same magnitude range as the real clusters.
We create the photometry for each star by linearly interpolating the isochrone grid (Parsec 1.1 from the Padova set) at the respective ages of the clusters and adding photometric uncertainties to the synthetic photometry that follow the same behavior as the observed errors. For all observed clusters we fitted an exponential curve to the photometric standard deviations as a function of the magnitude in each band. We then assigned every star an error that is drawn from a Gaussian distribution with a width that corresponds to the value of the exponential function at the respective magnitude plus a small random scatter comparable to the scatter of the real errors around the fitted curve. The upper panels of Fig. 5 show as an example the CMDs of four of the modeled clusters together with the theoretical isochrones that were used to create the photometry.

When comparing the artificial clusters with the observed ones (cf. Figs. 4 and 5) we note that the MS of the synthetic clusters is much broader at fainter magnitudes than the MS of the real clusters. This is due to the fact that we modeled the clusters using the photometric errors of the corresponding real clusters. If we look at Fig. 1 we see that the MS at fainter magnitudes is narrower than would be expected from the photometric errors. However, this does not affect our analysis as we do not use these regions in the CMD for the fitting of the SFH (see Sect. 4).

We carried out 100 Monte Carlo realizations of each cluster and fitted their SFH the same way as we did for the observed clusters (see Sect. 4). The results of the clusters that are shown as an example are presented in the lower panels of Fig. 5. The dots represent the mean contributed mass fraction that results from the 100 Monte Carlo realizations, at individual ages. The errorbars are the standard deviations. The (red) dashed line is the best Gaussian fit to the highest peak in the distribution, taking into account the errors. The thick vertical line at the $x$-axis marks the input age of the cluster. All fits reproduce the input age of the clusters very well within the errors. We note that the fits to the clusters show some additional low amplitudes of star formation at higher ages as it is observed in the real clusters (see Sect. 4). This is a first sign that these features are due to the fitting process and not intrinsic to the cluster itself. The fitted ages and standard deviations of all modeled clusters are summarized in Table 3 where we compare them with the results from the observed clusters. The dispersion of ages is lower than the spreads we found for the highest peak of most of the real clusters which suggest that the spread in the fitted SFH of the observed clusters is not only due to photometric errors. We will discuss this in Sect. 5.

\section{Results}

The aim of this work is to search for potential age spreads in a sample of eight young $(<1.1 \mathrm{Gyr})$ massive $\left(>10^{4} M_{\odot}\right)$ star clusters in the LMC. Our sample of clusters covers an age range from $20 \mathrm{Myr}$ to about $1 \mathrm{Gyr}$. The clusters analyzed in this work and in Bastian \& Silva-Villa (2013) have similar properties as the intermediate age (1-2 Gyr) LMC clusters that show extended or double MSTOs. The left panel of Fig. 6 shows the masses of the clusters of our sample as a function of the effective radius $R_{\text {eff }}$ along with the clusters presented in Goudfrooij et al. (2009, 2011a). Both, young and intermediate age clusters follow the same trend of increasing $R_{\text {eff }}$ with increasing mass. NGC 1847 is not included in this plot as it has a large uncertainty in its effective radius due to its shallow profile. The right panel of Fig. 6 shows the core radius $R_{\text {core }}$ as a function of logarithmic cluster mass for the same clusters. It is expected that, due to dynamical evolution, the spread in $R_{\text {core }}$ increases with the age of the 


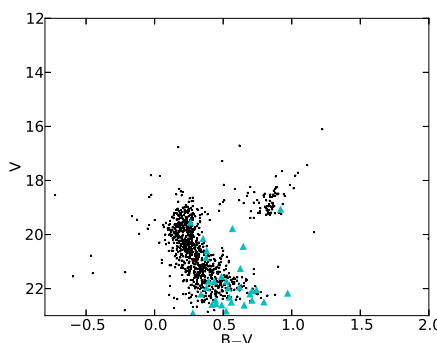

(a) NGC 2249

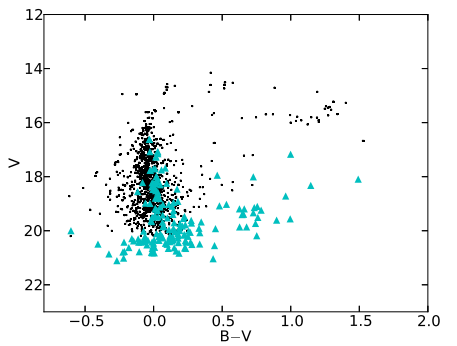

(e) NGC 1850

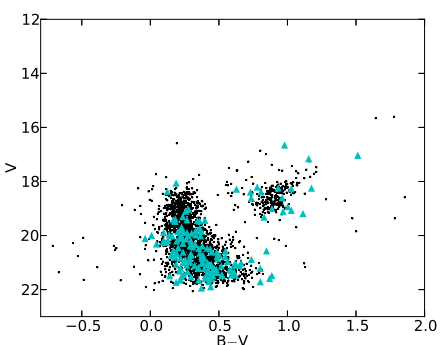

(b) NGC 1831

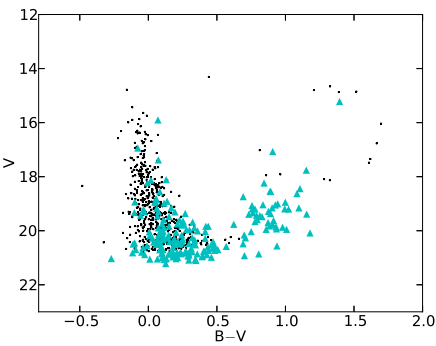

(f) NGC 1847

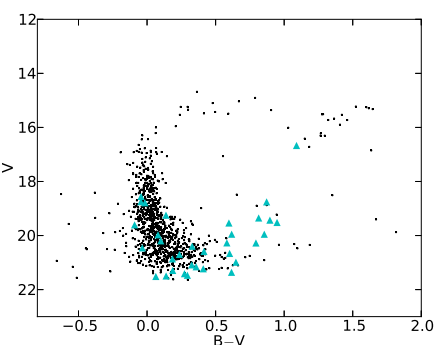

(c) NGC 2136

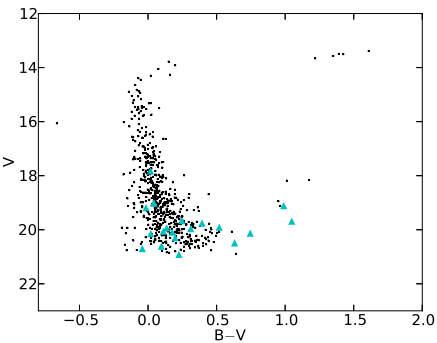

(g) NGC 2004

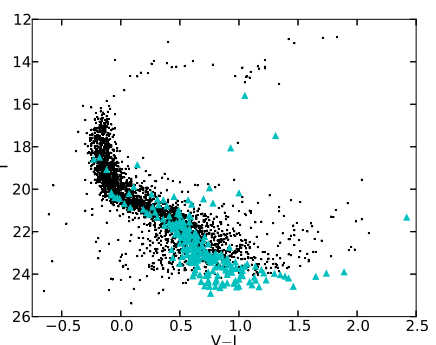

(d) NGC 2157

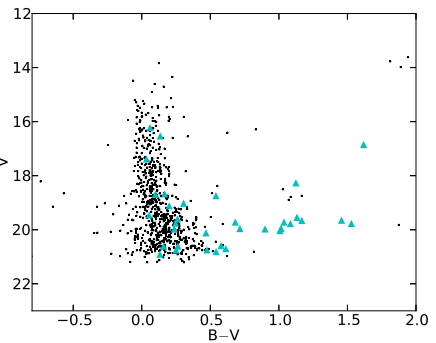

(h) NGC 2100

Fig. 4. CMDs of the clusters in our sample. The black dots are the stars that were used for the further analysis, whereas the (cyan) triangles indicate stars that were subtracted as field stars. Obviously, also some cluster stars were excluded in our SFH fits, but this does not affect our results. All CMDs contain only the stars in the inner regions of the clusters (two times the core radius). Note that the CMD of NGC 2157 is in the ( $V-I$ ) vs. $I$ space.

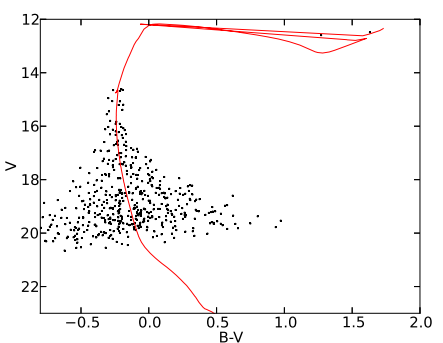

(a) $20 \operatorname{Myr}(B, V$ photometry)

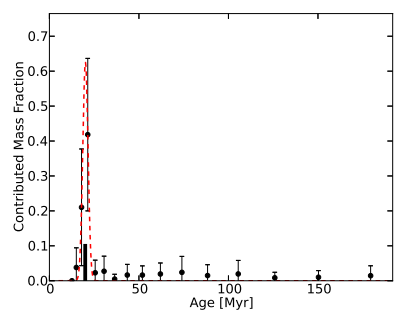

(e) $20 \operatorname{Myr}(B, V$ photometry)

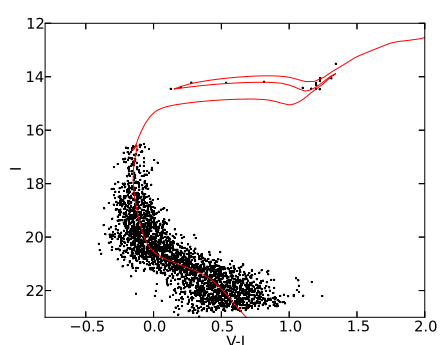

(b) $100 \mathrm{Myr}(V, I$ photometry)

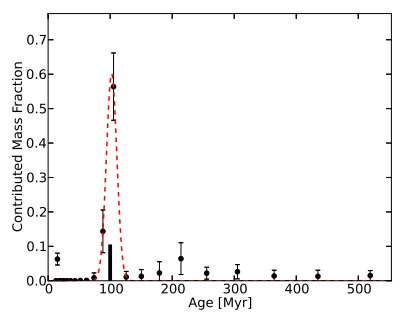

(f) $100 \operatorname{Myr}(V, I$ photometry)

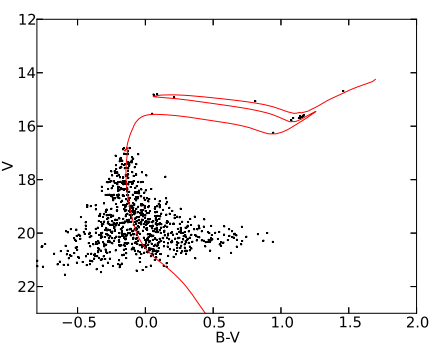

(c) $125 \operatorname{Myr}(B, V$ photometry)

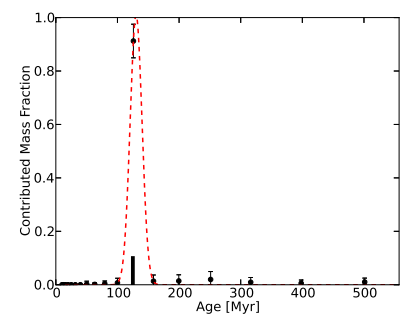

(g) $125 \operatorname{Myr}(B, V$ photometry)

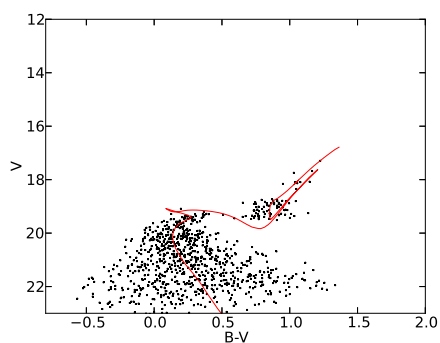

(d) $1.1 \mathrm{Gyr}(B, V$ photometry)

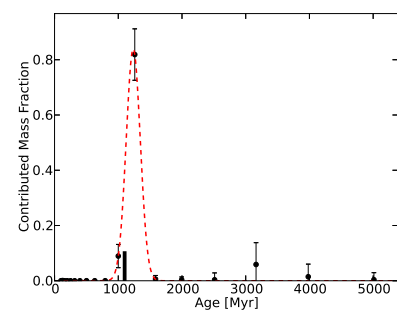

(h) $1.1 \mathrm{Gyr}(B, V$ photometry)

Fig. 5. Upper panels: CMDs of four of the artificial clusters that we created. We simulated these clusters to estimate the resulting age spread of a single-age population given by our SFH fitting code caused only by photometric errors. All stars in one cluster have the same age and the photometric errors are estimated from the ones of the real clusters at the respective age. The clusters shown here have ages of 20 , $100 \mathrm{Myr}$, $125 \mathrm{Myr}$ and $1.1 \mathrm{Gyr}$, spanning the range of ages of the real clusters in our sample. The (red) line is the theoretical Parsec 1.1 Padova isochrone at the respective age of the cluster. Lower panels: results of the SFH fits of the four artificial star clusters shown in the upper panel. The dots represent the mean contributed mass fraction at individual ages of the 100 realizations whereas the errorbars are the standard deviation. The dashed (red) line is the best-fit Gaussian to the highest peak of the points taking into account the errorbars. Indicated as a vertical think line at the $x$-axis is the actual age of the respective cluster. The fit to the $20 \mathrm{Myr}$ old cluster gives a peak at $20.1 \mathrm{Myr}$ with a dispersion of $1.5 \mathrm{Myr}$. We got an age of $102 \mathrm{Myr}$ for the $100 \mathrm{Myr}$ old cluster with an standard deviation of $8.4 \mathrm{Myr}$ and an age of $130 \mathrm{Myr}$ with a dispersion of $9.6 \mathrm{Myr}$ for the $125 \mathrm{Myr}$ old cluster. The $1.1 \mathrm{Gyr}$ old cluster has a fitted age of $1.2 \mathrm{Gyr}$. The standard deviation is $111 \mathrm{Myr}$.

clusters (e.g. Keller et al. 2011). We note that all the YMCs have systematically smaller core radii than the intermediate age clusters. One reason for this could be that the core radii given by Goudfrooij et al. (2009, 2011a) are constructed from the surface number density profiles, whereas McLaughlin \& van der Marel (2005) used surface brightness profiles. Both methods do not yield necessarily the same value for $R_{\text {core }}$. Due to dynamical mass segregation inside clusters the surface brightness profile 

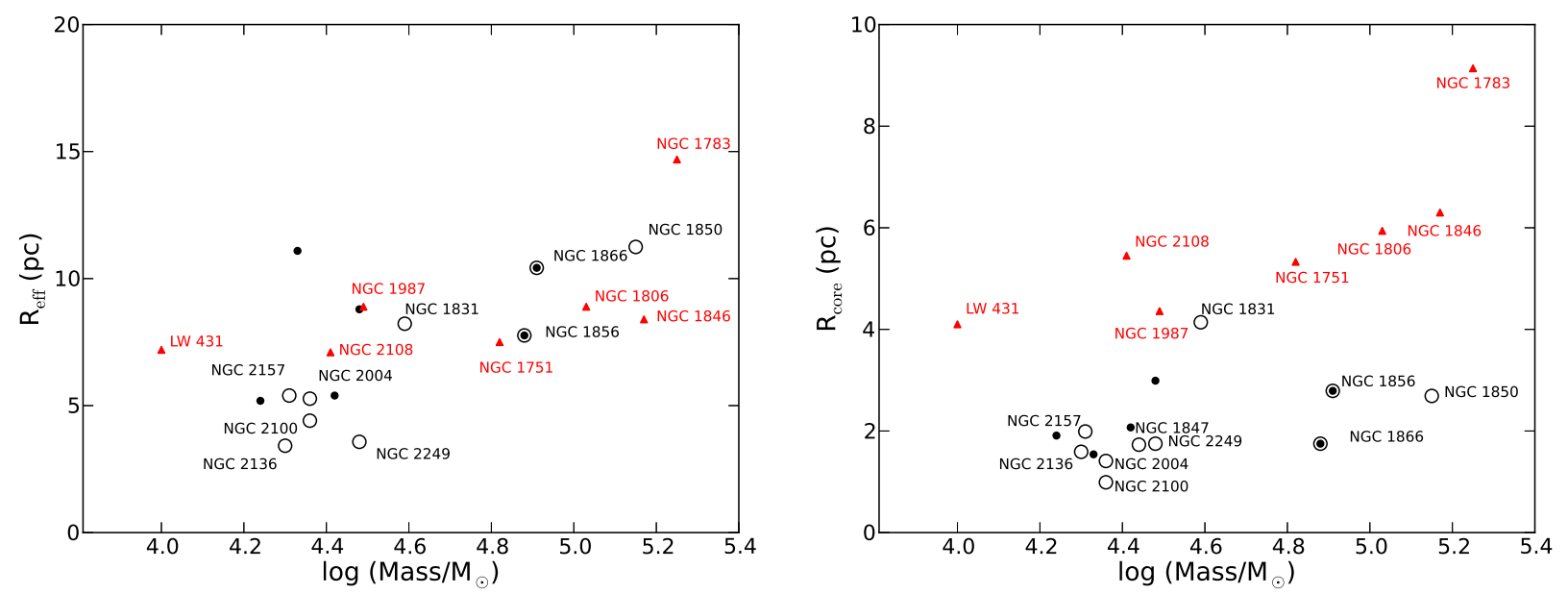

Fig. 6. Left panel: plot of the mass versus the effective radius for our sample of YMCs (black circles) and some additional clusters with similar properties (black dots), taken from the catalog of McLaughlin \& van der Marel (2005). The two clusters that are marked with a black dot surrounded by a black circle are the two clusters analyzed by Bastian \& Silva-Villa (2013). The (red) triangles are clusters of intermediate ages that display extended MSTOs, from Goudfrooij et al. (2009, 2011a). In this plot NGC 1847 is not shown as its effective radius has a large uncertainty due to its shallow profile. We increased the mass of NGC 1850 by a factor of 2 with respect to the literature value to account for the new age that we found (see Sect. 4.5). Right panel: same as the left panel but for the core radius. It is shown, e.g. in Keller et al. (2011), that the core radii of clusters have larger spreads the older the clusters are. We note here that the core radii of all clusters from the Goudfrooij et al. (2009, 2011a) sample are systematically higher than the ones from our sample. One reason for this could be that the core radii given in Goudfrooij et al. (2009, 2011a) are obtained from the surface number density, whereas McLaughlin \& van der Marel (2005) used the surface brightness profiles. Both methods do not yield the same value (see text).

Table 2. Regions in the CMDs of the clusters where the SFH was fitted.

\begin{tabular}{lccccc}
\hline \hline Cluster & $\begin{array}{c}\text { Color limits of the } \\
\text { blue fitting box }\end{array}$ & $\begin{array}{c}\text { Magnitude limits of the } \\
\text { blue fitting box }\end{array}$ & $\begin{array}{c}\text { Color limits of the } \\
\text { red fitting box }\end{array}$ & $\begin{array}{c}\text { Magnitude limits of the } \\
\text { red fitting box }\end{array}$ & $\begin{array}{c}\text { Number of stars } \\
\text { inside the boxes }\end{array}$ \\
\hline NGC 1831 & $-0.1 \leq(B-V) \leq 0.5$ & $-6.0 \leq V \leq 2.0$ & $0.5 \leq(B-V) \leq 1.4$ & $-6.0 \leq V \leq 1.5$ & 1015 \\
NGC 1847 & $-0.4 \leq(B-V) \leq 0.4$ & $-6.0 \leq V \leq 0.5$ & $0.4 \leq(B-V) \leq 1.5$ & $-6.0 \leq V \leq-2.5$ & 280 \\
NGC 1850 & $-0.3 \leq(B-V) \leq 0.1$ & $-6.0 \leq V \leq 0.0$ & $0.1 \leq(B-V) \leq 1.8$ & $-6.0 \leq V \leq-2.5$ & 995 \\
NGC 2004 & $-0.4 \leq(B-V) \leq 0.3$ & $-6.0 \leq V \leq 0.5$ & $0.3 \leq(B-V) \leq 1.7$ & $-6.0 \leq V \leq-1.5$ & 410 \\
NGC 2100 & $-0.4 \leq(B-V) \leq 0.2$ & $-6.0 \leq V \leq 0.0$ & $0.2 \leq(B-V) \leq 1.9$ & $-6.0 \leq V \leq-1.0$ & 375 \\
NGC 2136 & $-0.3 \leq(B-V) \leq 0.2$ & $-6.0 \leq V \leq 0.5$ & $0.2 \leq(B-V) \leq 1.7$ & $-6.0 \leq V \leq-0.5$ & 360 \\
NGC 2157 & $-0.3 \leq(V-I) \leq 0.2$ & $-6.0 \leq I \leq 1.5$ & $0.2 \leq(V-I) \leq 1.9$ & $-6.0 \leq I \leq-1.0$ & 1000 \\
NGC 2249 & $0.0 \leq(B-V) \leq 0.5$ & $-6.0 \leq V \leq 2.0$ & $0.5 \leq(B-V) \leq 1.7$ & $-6.0 \leq V \leq 1.5$ & 390 \\
\hline
\end{tabular}

is more concentrated towards the center and therefore results in a smaller value for the core radius.

We fitted theoretical isochrones from the Parsec 1.1 isochrone set (Bressan et al. 2012) to the observed CMDs to estimate the clusters' metallicity, distance modulus and the reddening towards the clusters. This set of isochrones uses a value of 0.0152 for the solar metallicity $Z_{\odot}$. Table 1 lists the basic properties of the LMC clusters that are the subject of this work. For the further analysis we dereddened the magnitudes of all stars in each cluster by the same value of the derived reddening (we assume no differential reddening, except for NGC 2100). The data of NGC 2157 is already corrected for a reddening value of $E(B-V)=0.1$ by Fischer et al. (1998).

As a second step we fitted the SFH of each cluster using the code FITSFH (Silva-Villa \& Larsen 2010). For the fitting we used the previously determined metallicity and distance and assumed a Salpeter (1955) IMF.

We performed the fitting in two regions (a "blue" and a "red" fitting box) of the CMD for each cluster. The blue box contains the MS of the cluster whereas the red box covers the regions of the evolved stars. The limits of all the boxes and the total number of stars in those boxes are summarized in Table 2. The faint limits of the boxes containing the MS are chosen such that they are at least 0.5 mag brighter than the $90 \%$ completeness limits. We did several fits with different choices of the box limits. Thereby we noticed that the overall result does not depend on the exact choice of the boxes. To assess the statistical errors of the fittings that result from the stochastic IMF population of stars we performed additional bootstrapping tests. We created for each cluster 50 bootstrap samples and ran the SFH for each of these samples. The figures of the SFH fits presented in the next subsections show the mean values at individual ages (black dots) and the one sigma errorbars that follow from the bootstrapping procedure.

The results of the SFH fitting will give us upper limits of potential age spreads as we do not take into account binaries and differential reddening.

In the following we present the age and SFH fitting results of each cluster in order of decreasing age.

\subsection{NGC 2249}

NGC 2249 is the oldest cluster in our sample. The literature age of the cluster is between 660 Myr (Baumgardt et al. 2013) and about 1 Gyr (e.g. Kerber et al. 2007; Correnti et al. 2014). It is 

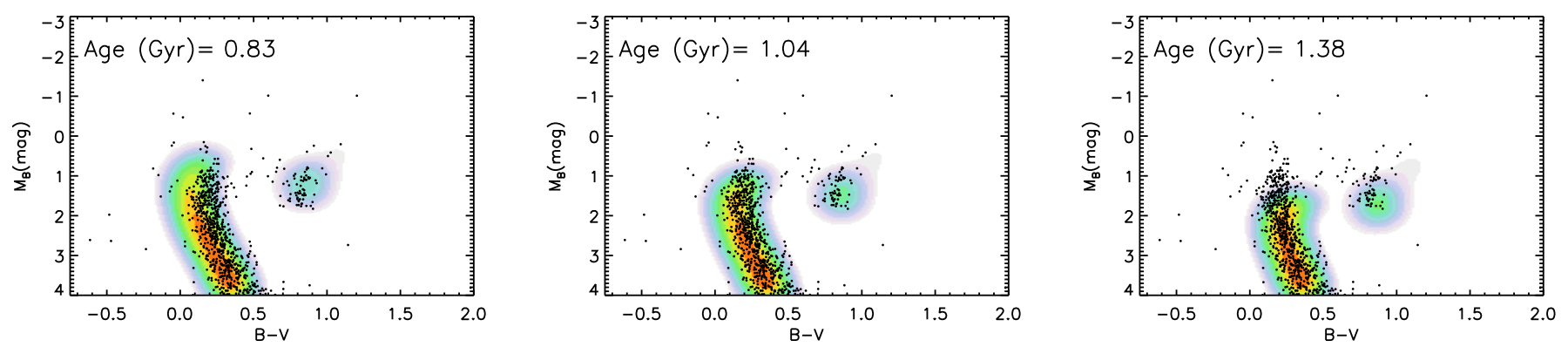

Fig. 7. CMD of NGC 2249 with overlaid model Hess diagrams at three different ages. The black dots are the individual observed stars and the filled (colored) contours are theoretical Hess diagrams. Their respective ages are indicated in the upper left corner of each panel.

therefore not a YMC any more but rather belongs to the category of intermediate age LMC clusters. Adopting an age of 1 Gyr, Correnti et al. (2014) found a mass of $3.0 \times 10^{4} M_{\odot}$ for this cluster. We matched theoretical isochrones to NGC 2249 to determine its basic parameters for the further analysis. We found a reddening $E(B-V)$ of 0.02 , a metallicity of $Z=0.008$ and a DM $(m-M)_{0}$ of $18.3 \mathrm{mag}$. These values are in good agreement with previous studies. Kerber et al. (2007), who also used the Brocato et al. (2001) data set, modeled the CMDs of various clusters using Padova isochrones from Girardi et al. (2002). For NGC 2249 they find a value of 0.01 for the reddening, a metallicity $Z$ of 0.007 and a DM of $18.27 \mathrm{mag}$. The recent study by Correnti et al. (2014) that used deep HST photometry finds similar values fitting Marigo et al. (2008) isochrones to the CMDs $\left(E(B-V)=0.02,(m-M)_{0}=18.2 \mathrm{mag}, Z=0.006\right)$.

Figure 7 shows the CMD of NGC 2249. The black dots indicate individual observed stars. The CMD shows two evident features: the MS of the cluster which extends up to a $B$ band magnitude of $\sim 0.8$ and the red clump at $B \sim 1.3$ mag and $B-V \sim 0.8$. Overplotted are model Hess diagrams at three different ages from $830 \mathrm{Myr}$ to $1.4 \mathrm{Gyr}$ (filled contours). The position of the red clump in the CMD and the MSTO is best reproduced by an age of about $1 \mathrm{Gyr}$, in agreement with the findings by Kerber et al. (2007) and Correnti et al. (2014). At an age of $830 \mathrm{Myr}$ the MS extends to brighter magnitudes than it is observed and at $1.4 \mathrm{Gyr}$ the models predict a MSTO that is fainter than observed.

For a more quantitative analysis of the cluster age we fit the SFH of the cluster using the FITSFH code (Silva-Villa \& Larsen 2010) providing the observed magnitudes and photometric errors of the stars as input. We did the fit of the SFH in two regions in the CMD. The limits of the chosen boxes can be found in Table 2. The blue fitting box contains the MS down to a magnitude of 2.0 which is about half a magnitude above the $90 \%$ completeness limit given by Brocato et al. (2001). The red fitting box covers the red clump stars. Figure 8 shows the results of the SFH fit. The points are the mean contributed mass fraction of the fit at individual ages with one sigma errorbars that result from the bootstrapping. The solid line is the best fit Gaussian taking into account the errorbars. The curve peaks at an age of $\sim 1.11 \mathrm{Gyr}$. The dispersion of $139 \mathrm{Myr}$ is a measure of the maximum age spread that is present in the cluster.

\subsection{NGC 1831}

NGC 1831 is the second oldest cluster in our sample. Kerber et al. (2007) found an age of $700 \mathrm{Myr}$ for this cluster, a metallicity $Z$ of 0.016 , an extinction $E(B-V)$ of 0.01 and a DM of 18.23. Li et al. (2014) analyzed the CMD of NGC 1831 and found that the MSTO is broader than expected. They conclude that this

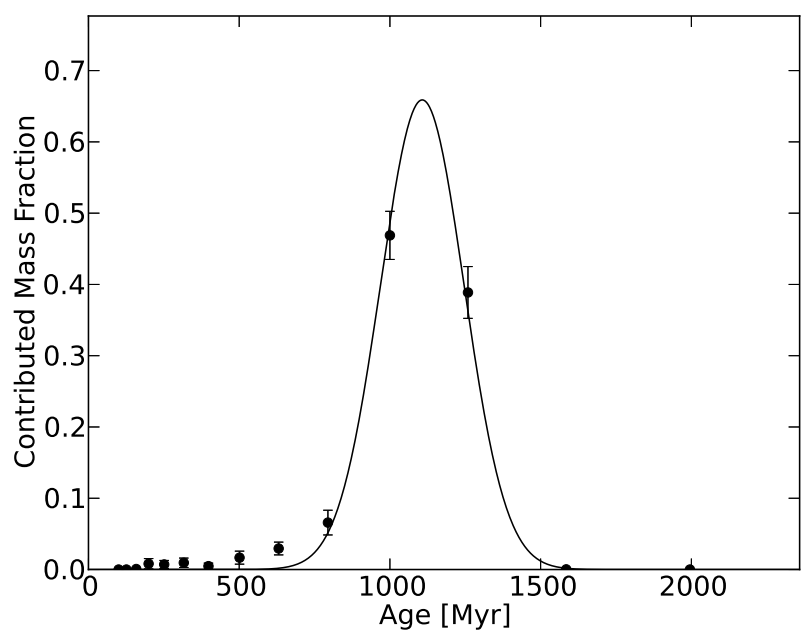

Fig. 8. Results of the SFH fit of NGC 2249. The dots represent the contributed mass fraction at individual ages and the solid line shows the best Gaussian fit to the points with a peak at $1.11 \mathrm{Gyr}$ and a standard deviation of $139 \mathrm{Myr}$.

feature is best explained with stars of different rotation velocities and a dispersion of ages between 550 and 650 Myr. For their analysis they used the following parameters: $Z=0.012$, $E(B-V)=0.03$ and $(m-M)_{0}=18.4$ mag. We determined an extinction $E(B-V)$ of 0.0 , a metallicity $Z$ of 0.016 and a distance modulus of $18.1 \mathrm{mag}$ that we used for the analysis of the SFH of NGC 1831. The CMD of the cluster with superimposed model Hess diagrams is shown in Fig. 9. It is very similar to the CMD of NGC 2249 suggesting that both clusters have comparable ages. The MS of NGC 1831 extends up to $M_{B} \sim 0.5 \mathrm{mag}$. The position of the red clump is at a $B$ magnitude of about 1.2 and a $B-V$ color of $\sim 0.9$. Looking at the Hess diagrams we see that the model at 870 Myr fits best the data.

We fitted the SFH of the cluster in two regions in the CMD. The limits of the fitting boxes are given in Table 2. The boxes contain the MS as well as the He-burning stars. The results of the fitting are shown in Fig. 10. A Gaussian fit to the individual points that represent the contributed mass fraction at single ages gives a peak at an age of $924 \mathrm{Myr}$. The standard deviation of the Gaussian (126 Myr) is the upper limit of the age spread as we do not consider other effects like binaries, rotating stars or differential extinction that might effect the CMD.

\subsection{NGC 2136}

NGC 2136 is younger than the previous two clusters. Its age from the literature is about $100 \mathrm{Myr}$ (e.g. Dirsch et al. 2000). By 

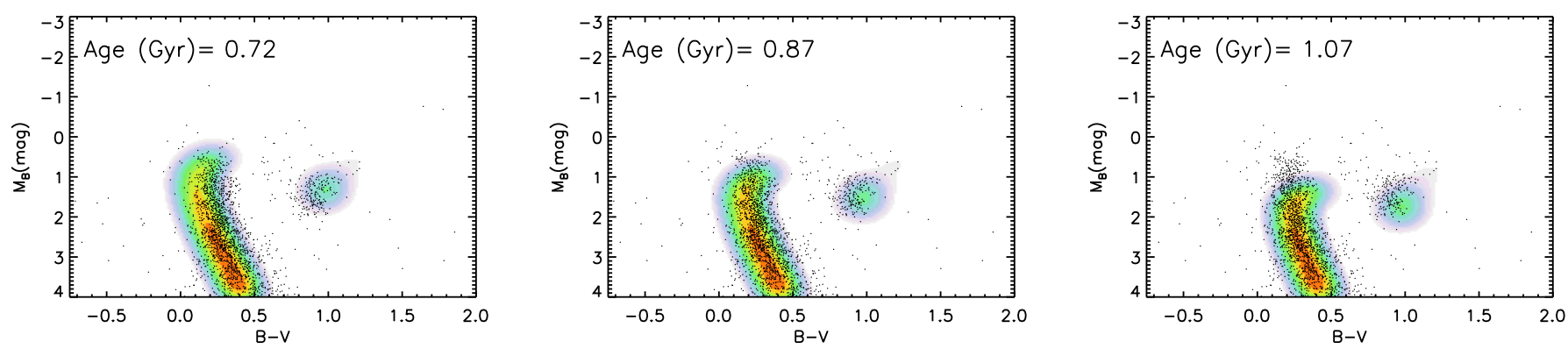

Fig. 9. CMD of NGC 1831 with overlaid model Hess diagrams at three different ages from 750 Myr to 1.04 Gyr.

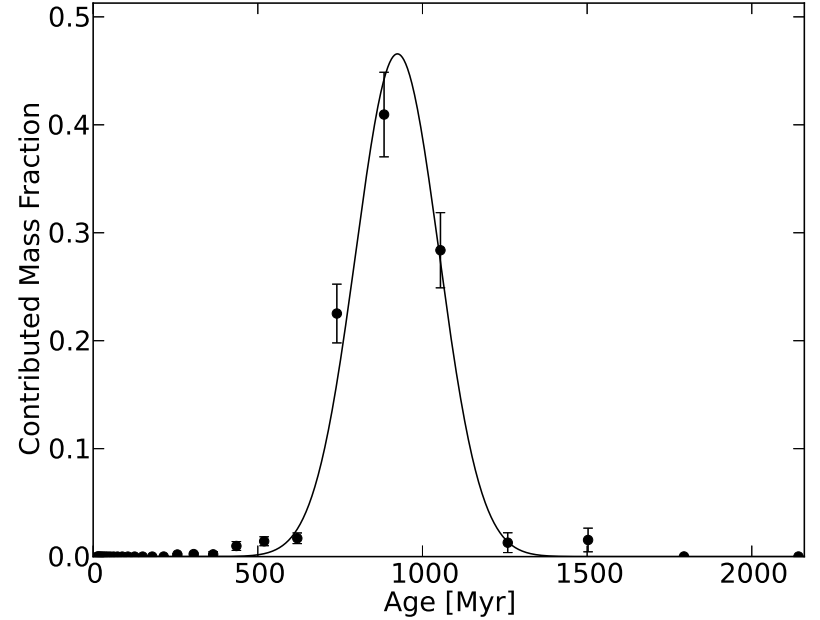

Fig. 10. Results for the fitting of the SFH of NGC 1831. The dots represent the results at individual ages and the solid line shows the best Gaussian fit to the points with a peak at $924 \mathrm{Myr}$ and a standard deviation of $126 \mathrm{Myr}$.

overplotting isochrones onto the cluster's CMD we find a reddening $E(B-V)$ of 0.13 , which is in agreement with the value of $0.1 \pm 0.03$ found by Dirsch et al. (2000). Furthermore, we determined the metallicity $Z$ to be 0.005 which is somewhat higher than the value of 0.004 by Dirsch et al. (2000) and a distance modulus of 18.5 mag that is consistent with the mean distance to the LMC (de Grijs et al. 2014). Figure 11 shows the CMD of NGC 2136 overlaid with Hess diagrams at ages of 100, 125 and 177 Myr. The top of the MS in this cluster is at $B \sim-2$ followed by an almost continuous sequence of already evolved stars which reaches a top brightness of $M_{B} \sim-4$ and extends to a $B-V$ color of $\sim 1.5$. We note that there are stars in the so called "Blue-Hertzsprung-Gap" between the MS and the subgiant branch where no stars are predicted by the models. These stars could be stars in the dense cluster center that are affected by crowding, fast rotating stars, interactive binaries or higher mass stars that formed out of the merging or collision of two low mass stars ("blue stragglers"). The blue loop region in the CMD that is populated by He-burning stars is well sampled in this cluster which makes it easier to constrain a possible age spread just by looking at the theoretical Hess diagrams. We see that an age of $125 \mathrm{Myr}$ fits best the position of the evolved stars, especially the red side of the blue loop (Fig. 11 middle). Also an age of $100 \mathrm{Myr}$ is compatible with the data, although the model seems to be a bit too bright (Fig. 11 left). On the other hand, an age of $\sim 180 \mathrm{Myr}$ is too old for NGC 2136 (Fig. 11). The theoretical Hess diagram fails to fit the blue loop stars as well as the position of the MS turn off. From this we can already rule out a possible age spread of more than $\pm 50 \mathrm{Myr}$.
To put our initial estimate on a more quantitative basis we fitted the SFH using FITSFH, as we did in the previous clusters. We adopted two fitting boxes that were chosen such that they include the main features of the cluster CMD but exclude the leftover contamination of faint red field stars (compare Fig. 4). The faint limit of the blue fitting box is one magnitude above the $90 \%$ completion limit given by Brocato et al. (2001). The fitting of the SFH of NGC 2136 gives a period of star formation which peaks at an age of $\sim 123 \mathrm{Myr}$ with an upper limit of \pm 23 Myr for its duration (see Fig. 12), comparable with our first estimate. We also note, that there is an additional significant peak at 200 Myr. Figure 13 shows a Hess diagram at $200 \mathrm{Myr}$ superimposed over the CMD of NGC 2136. The fitted star formation at $200 \mathrm{Myr}$ is due to the faintest stars at the red end of the blue loop at $B-V \sim 1.0$. But at this age we would also expect a higher density of blue loop stars at a $B-V$ color of about 0.4 and a $B$ magnitude of about -2.5 where no stars are observed. Therefore, we can rule out the peak at $200 \mathrm{Myr}$.

\subsection{NGC 2157}

NGC 2157 is not part of the Brocato et al. (2001) data set and its data is taken from Fischer et al. (1998) who provides photometry in the $V$ and $I$ filters. The photometry is already corrected for extinction by Fischer et al. (1998) who adopted a reddening value $E(B-V)$ of 0.1 . We derived the same value and therefore did not change it for our analysis. By plotting theoretical isochrones over the observed CMD of the cluster they determined an age of $100 \mathrm{Myr}$ for the cluster. We estimated a metallicity $Z$ of 0.008 and a distance modulus of $18.5 \mathrm{mag}$ for the cluster, consistent with the values assumed for the cluster by Fischer et al. (1998). Figure 14 shows the CMD of the cluster together with theoretical Hess diagrams overplotted. As NGC 2136, which has a similar age, the CMD of NGC 2157 shows a clear MS turn off at about $M_{V}=-3$ and a well populated blue loop with a slight overdensity of stars at $V-I \sim 1.2$ that marks the red envelope of the loop. In agreement with Fischer et al. (1998) we note that an age of 100 Myr fits best the observed stellar distribution in the CMD (Fig. 14 middle). From the CMD we can constrain an age spread to be less than about $\pm 30 \mathrm{Myr}$.

We chose the regions in the CMD that we used to fit the SFH of the cluster such that they contain the main features of the cluster plus some additional space below and above the sequence of evolved stars to better constrain younger and older ages (see Table 2 for the exact limits). The results of the fitting procedure are displayed in Fig. 15. As expected, the star formation has a clear maximum at about $100 \mathrm{Myr}$ with a upper limit of an age spread of \pm 13.2 Myr. Additionally, the fit yields a high value of star formation at $50 \mathrm{Myr}$ with a $1.5 \sigma$ significance. This age corresponds to the stars that are at the top of the MS at $M_{V}=-3$. 

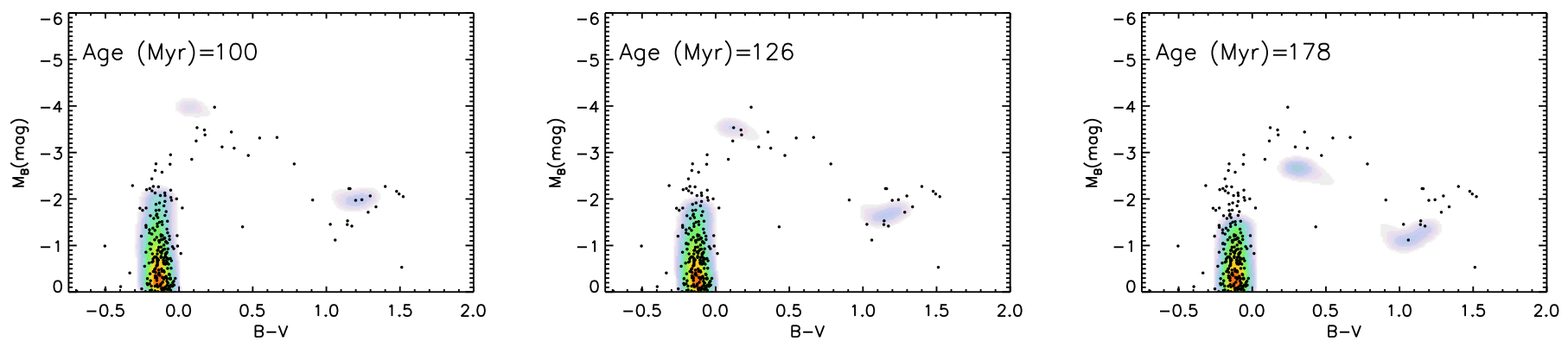

Fig. 11. CMD of NGC 2136 with overplotted theoretical Hess diagrams at 100, 126 and 178 Myr.

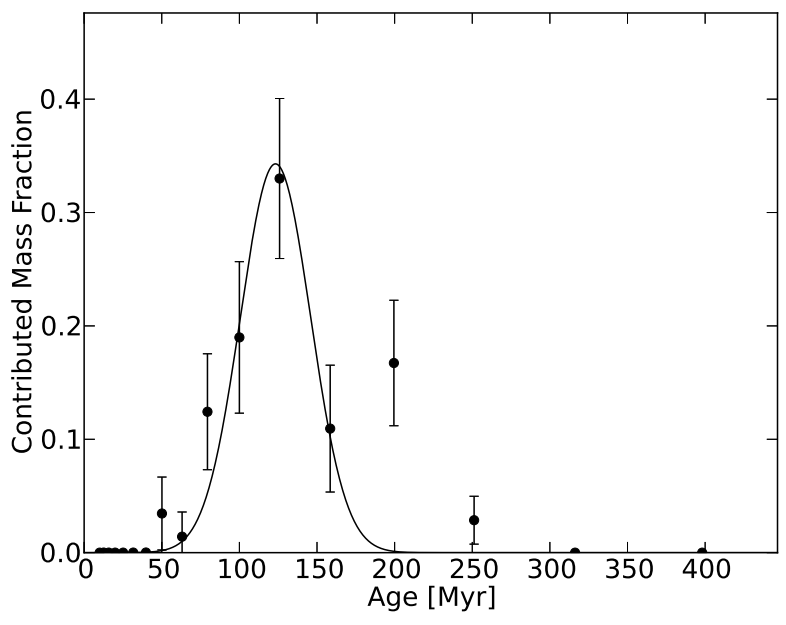

Fig. 12. Results of the SFH fit of NGC 2136. The dots represent the results at individual ages and the solid line shows the best Gaussian fit to the points with a peak at $123.3 \mathrm{Myr}$ and a standard deviation of $22.6 \mathrm{Myr}$.

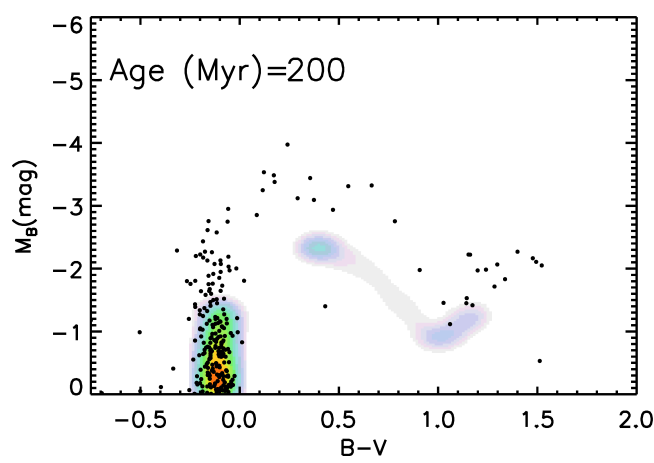

Fig. 13. CMD of NGC 2136 with an overplotted theoretical Hess diagram at $200 \mathrm{Myr}$.

However, the position of the evolved stars at $50 \mathrm{Myr}$ do not agree with the observations. Therefore this age can be excluded.

\subsection{NGC 1850}

NGC 1850 is a binary cluster system consisting of a main cluster (NGC 1850A) and a small and dense secondary cluster (NGC 1850B) composed mainly of young O and B stars. This cluster is the only one for which we obtained the data from the HST Legacy Archive. The data catalog consists of the spatial positions of the stars on the detector chip, the RA and Dec coordinates and the $\mathrm{AB}$ magnitudes in the $F 450 \mathrm{~W}$ and $F 555 \mathrm{~W}$ filter, however without photometric errors. We converted the magnitudes to Vega magnitudes and afterwards to the Johnson $B V$ system and created artificial photometric errors that follow the same behavior as the observed ones in the Brocato et al. (2001) data set of the other clusters. In our study we consider only the central parts of the main cluster NGC 1850A (all stars within two times the core radius). The young stars of the secondary cluster are outside this area and therefore they will not affect our analysis. We determined a reddening $E(B-V)$ of 0.1 which is smaller than the value of 0.17 that is assumed by Fischer et al. (1993). Additionally, a metallicity of $Z=0.006$ and a distance modulus of $18.5 \mathrm{mag}$ reproduces best the position and extent of the blue loop. The distance modulus is a bit smaller than $18.6 \mathrm{mag}$ that was determined by Sebo \& Wood (1995) by measurements of Cepheids. The resulting CMD of NGC 1850A is shown in Fig. 16. It shows a MS that extends up to a $B$ magnitude of about -3.5 and a track of evolved blue loop stars that goes to a $B-V$ color of about 1.3. Comparing the observed CMD with the model we see that for an age of about 90 Myr the best accordance is found. The magnitude and the morphology of the evolved stars matches the observations best at this age. We can exclude ages older than $125 \mathrm{Myr}$ and younger than about $70 \mathrm{Myr}$. We note that also in this cluster the MS extends to brighter magnitudes as would be expected from the models.

We fitted the SFH in two regions in the CMD that contain the MS and the post-MS track (see Table 2). As already mentioned at the end of Sect. 2, we made a conservative estimate of the completeness limit (18.5 mag in the $V$ band) of NGC 1850 and chose the limits of the fitting boxes according to that. The results of the fitting is displayed in Fig. 17. We find a distribution that peaks at an age of about $93 \mathrm{Myr}$ with a maximum spread of $18 \mathrm{Myr}$. This is in agreement with the result by Fischer et al. (1993) who found an age of $90 \pm 30 \mathrm{Myr}$. However, besides the main peak at $93 \mathrm{Myr}$ the fitting of the SFH gives a considerable amount of stars that formed at ages between 200 and $400 \mathrm{Myr}$. The lower three panels of Fig. 16 show the CMD of NGC 1850 with superimposed Hess diagrams at ages of 200, 300 and 400 Myr. At these older ages there are no evolved stars observed where the models would predict them. As an additional test to rule out the fitted ages of a few hundreds of Myrs, we created an artificial cluster with the fitted SFH of NGC 1850, normalized to the total number of stars in NGC 1850 brighter than an absolute magnitude of 0.0 in the $V$ band. We chose this limit to be sufficiently bright in order to avoid any effects of incompleteness. The result is shown in Fig. 18. In this figure, the stars are color-coded by their ages, whereas the youngest stars are black and the oldest are white. From the synthetic cluster we expect stars to be between 0.7 and 1.2 in $B-V$ color and between -2 and 0.5 in $B$ magnitude. However, there are no stars found in this region of the CMD of NGC 1850. We can therefore rule out 

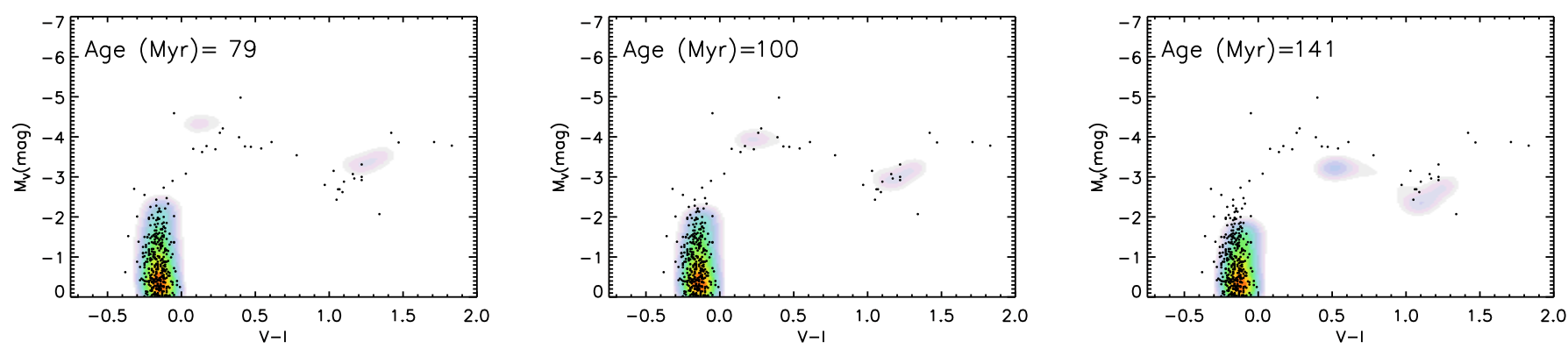

Fig. 14. CMD of NGC 2157 with overlaid Hess diagrams at the ages $80 \mathrm{Myr}, 100 \mathrm{Myr}$ and $140 \mathrm{Myr}$. Note that this CMD, in contrast to the other cluster CMDs, is in the $(V-I)$ vs. $M_{V}$ space.

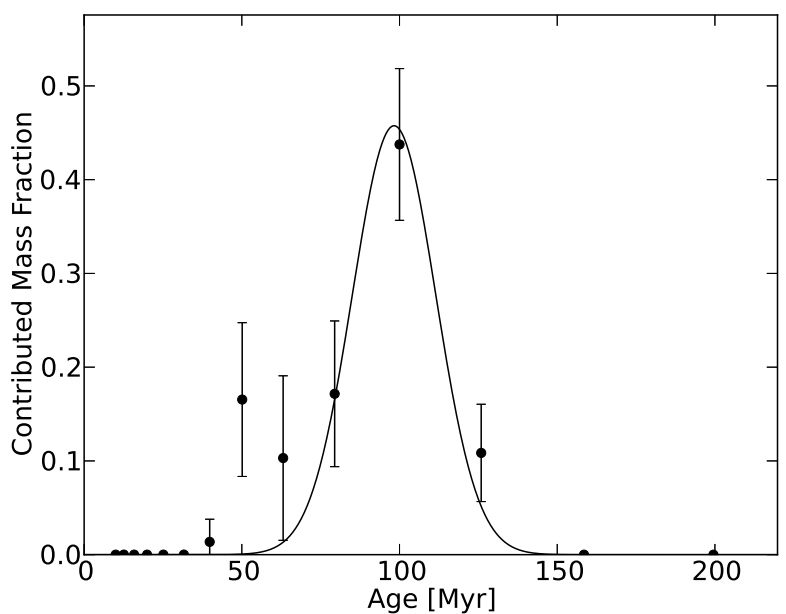

Fig. 15. Results of the SFH fit of NGC 2157. The dots represent the results at individual ages and the solid line shows the best Gaussian fit to the points with a peak at $98.3 \mathrm{Myr}$ and a standard deviation of 13.2 Myr.

stars that have ages older than 200 Myr. An alternative explanation of these older ages could be contributions from field stars as NGC 1850 has a high level of field star contamination (see Fig. 4).

The age that we found for NGC 1850A is significantly higher than the widely used value of $30 \mathrm{Myr}$ in the literature (e.g. Elson 1991; McLaughlin \& van der Marel 2005; Baumgardt et al. 2013). Adopting this new age of about $100 \mathrm{Myr}$ results in a mass that is twice as large as the one that is estimated from an age of $30 \mathrm{Myr}$. So, the new mass of NGC 1850 is $1.4 \times 10^{5} M_{\odot}$ instead of $7.2 \times 10^{4} M_{\odot}$. This new mass is consistent with the expected mass-to-light ratio for a $100 \mathrm{Myr}$ population (McLaughlin \& van der Marel 2005), which further supports our result.

\subsection{NGC 1847}

NGC 1847 is one of the younger clusters in our sample. Elson \& Fall (1988) dated this cluster to an age of $26 \mathrm{Myr}$. We find a reddening $E(B-V)$ of 0.16 which is somewhat higher than the value by Nelson $\&$ Hodge (1983) who adopted $E(B-V)=0.1$ for their studies and a metallicity $Z$ of 0.006 , in agreement with Mackey \& Gilmore (2003).

According to McLaughlin \& van der Marel (2005) NGC 1847 has an effective radius of more than 30 pc (assuming King 1966 profiles). This is more than 4 times as large as it would be expected from its mass assuming it follows the same $R_{\text {eff }}-\log (M)$ relation as the other young and intermediate age LMC clusters (see Fig. 6). However, NGC 1847 has a very shallow density profile (e.g. Mackey \& Gilmore 2003) and therefore the value of $R_{\text {eff }}$ is afflicted with a large uncertainty.

Figure 4f shows the CMD of the central part of NGC 1847. The black dots are the cluster stars used for the analysis and the red crosses are the stars subtracted as field stars. Beneath the cluster population there is a second numerous population of stars at older ages (about $800 \mathrm{Myr}$, corresponding to the position of the red clump) that is not present in such an extent in the other CMDs. This population is most likely due to the background LMC stars, as NGC 1847 is close to the LMC bar.

Figure 19 shows model Hess diagrams at different ages overlaid over the CMD of NGC 1847. An age of about 56 Myr reproduces best the position of the He-burning stars. On the one hand, there are no evolved stars that would be suggestive of ages older than 70 Myr. On the other hand, at 56 Myr the observed MS extends beyond the model by more than one magnitude. Only ages of about 20 to $25 \mathrm{Myr}$ can account for these stars. By just comparing the CMD with the models we can restrict the ages to be between 20 and $70 \mathrm{Myr}$.

In the fitting of the SFH we include the regions in the CMD that contain the MS down to a $V$ magnitude of 0.5 and the red He-burning stars. The results of the fitting (Fig. 20) show a large peak at $57 \mathrm{Myr}$ and an irregularly shaped lower star formation at ages between 14 and $30 \mathrm{Myr}$. The result at younger ages is due to the MS extending to brighter magnitudes than would be expected for a $57 \mathrm{Myr}$ old population. There is no sign for this younger population in the post-MS part of the CMD. This might be due to the random sampling of the IMF. Therefore, to evaluate the results of the SFH fit statistically, we create 1000 synthetic clusters with the same SFH as we got for NGC 1847 normalized to the number of stars present in the CMD of NGC 1847 brighter than a $V$ band magnitude of 0.5 to avoid effects resulting from incompleteness. Figure 21 shows the CMD of one of the clusters where the individual stars are color-coded by their age. We analyzed the occurrence of post-MS stars of all individual ages to get a measure of how many stars we should expect at a given age (see Fig. 22). A density plot of the post-MS tracks of all 1000 clusters stacked together is shown in Fig. 23 to visualize where in the CMD the stars are expected to be. We see from Fig. 22 that in a considerable fraction of cases $(82 \%$ at an age of $25 \mathrm{Myr}$ and $40 \%$ at an age of $30 \mathrm{Myr}$ ) there are no post-MS stars present at ages where the young peak is. So from a statistical point of view we cannot rule out the second peak at 14-30 Myr. If the star formation at the younger ages is real, this would result in an overall total age spread of $<45 \mathrm{Myr}$ which is inconsistent with the models proposed by Goudfrooij et al. (2009, 2011a,b) and Rubele et al. (2013).

However, there might be other causes for the bright MS stars that are suggestive of a 26 Myr old population: they could be binaries, blue straggler stars or fast rotating stars. In an upcoming 

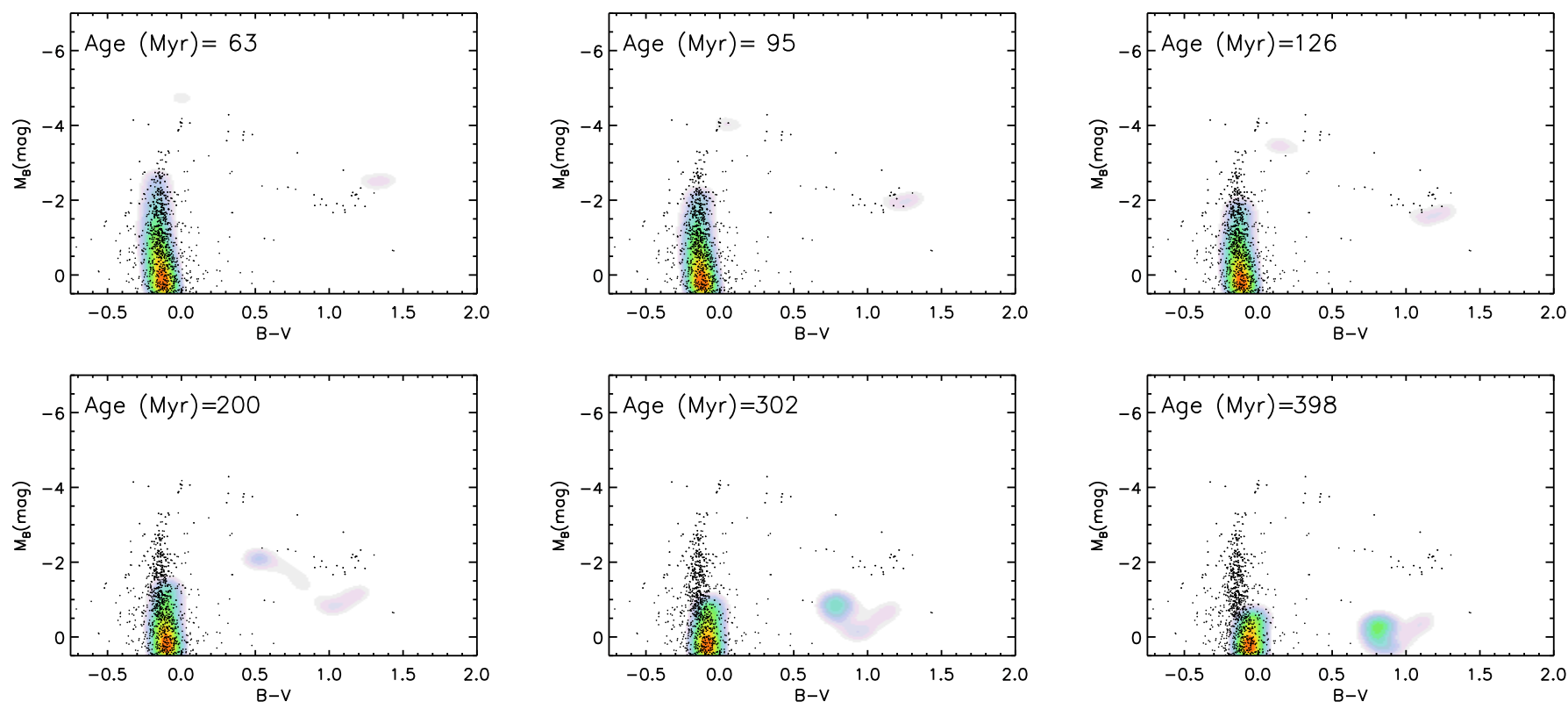

Fig. 16. CMD of NGC 1850 with superimposed model Hess diagrams at ages from 60 to $400 \mathrm{Myr}$.

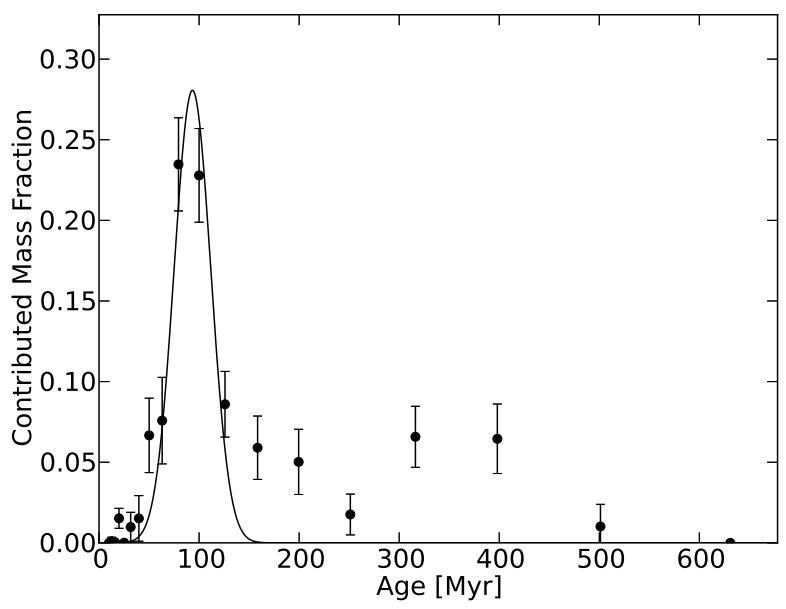

Fig. 17. Results for the fitting of the SFH of NGC 1850. The dots represent the results at individual ages and the solid line shows the best Gaussian fit to the points with a peak at $93.4 \mathrm{Myr}$ and a standard deviation of 18.3 Myr.

paper (Niederhofer et al., in prep.) we will investigate the role of rotation and test the last hypothesis.

\subsection{NGC 2004}

NGC 2004 also belongs to the youngest group of clusters in our YMC sample. Elson (1991) found an age of $20 \mathrm{Myr}$ for this cluster. It has a total mass of $\sim 2.3 \times 10^{4} M_{\odot}$ (McLaughlin \& van der Marel 2005). By matching theoretical isochrones to the MS of the cluster we find a reddening value of $E(B-V)=0.23$ which is considerably higher than the value of 0.08 found by Keller et al. (2000). Figure 24 shows the CMD of this cluster together with Hess diagrams at three different ages. The observed CMD consists of two main features: the MS that extends to magnitudes of about -5 in the $B$ band and a handful of red He-burning stars at $(B-V) \sim 1.2$. The position of this Heburning stars is best reproduced by an age of 20 Myr (Fig. 24 middle panel). Ages that are older than about 30 Myr can be excluded because there are no He-burning stars at the position in

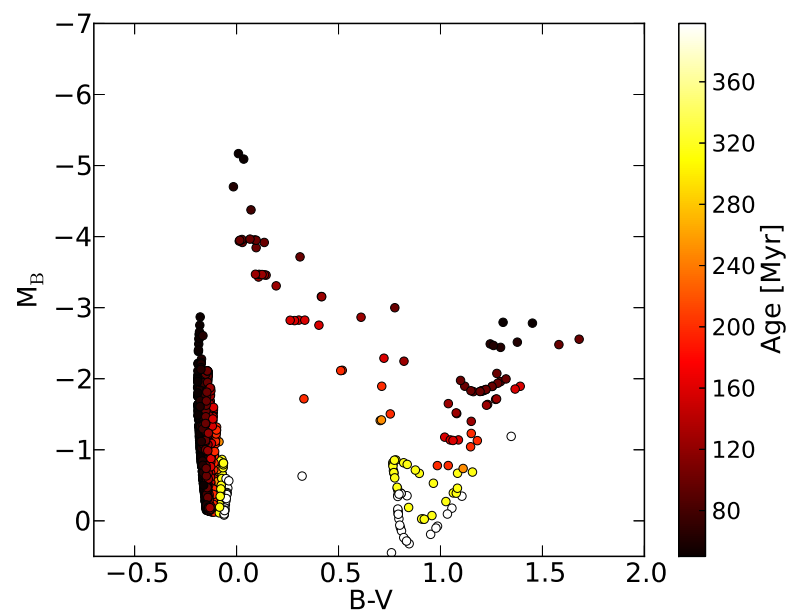

Fig. 18. CMD of an artificial cluster created using the fitted SFH of NGC 1850 normalized to the number of stars present in the used data set of NGC 1850 that are brighter than 0.0 mag in the $V$ band. The stars are color-coded by their ages going from black (the youngest) to white (the oldest stars).

the CMD predicted by the models. Younger models do not show any evolved stars. We note that the observed MS for all ages extends to brighter magnitudes than is predicted by the theoretical model. This might be caused by the used set of isochrones as the Parsec 1.1 isochrones only include masses up to $12 M_{\odot}$. At an age of $\sim 18 \mathrm{Myr}$, the turn-off mass is about $10.4 M_{\odot}$ so the left panel of Fig. 24 shows only the MS and no stars that already evolved off the MS. For a comparison we show in Fig. 25 the CMD of NGC 2004 with Hess diagrams constructed using Padova isochrones from the Marigo et al. (2008) set which contain models for stars with masses up to $100 M_{\odot}$. The age which matches best the helium burning stars is about $22 \mathrm{Myr}$ and therefore a bit older than the one from the Parsec 1.1 isochrones. But also this model cannot explain the bright stars that extend over the MS at this age. In order to explain the bright MS stars an age of 12.5 Myr for the cluster is needed (see Fig. 25). However, the position of the He-burning stars at this age does not correspond 

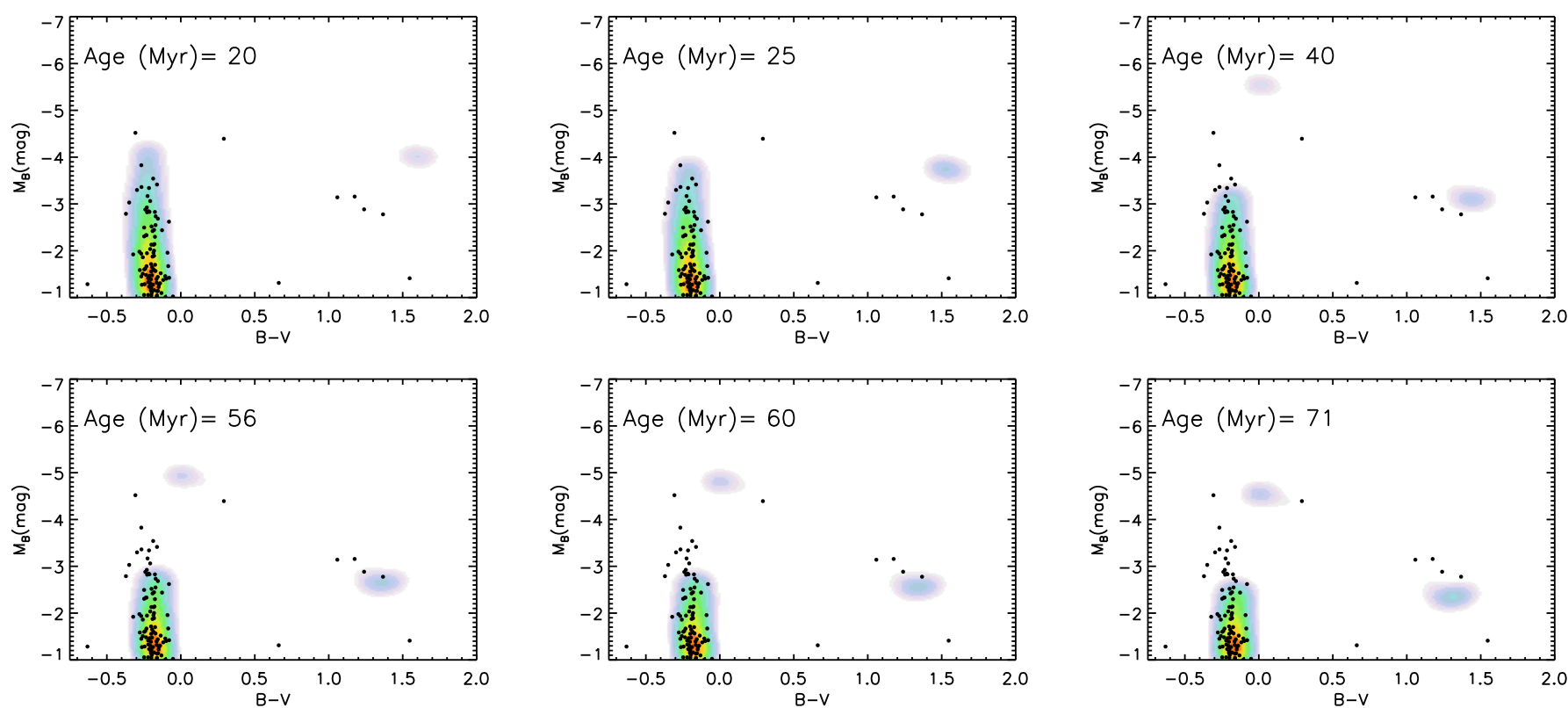

Fig. 19. CMD of NGC 1847 with overlaid model Hess diagrams at six different ages ranging from 20 to 70 Myr.

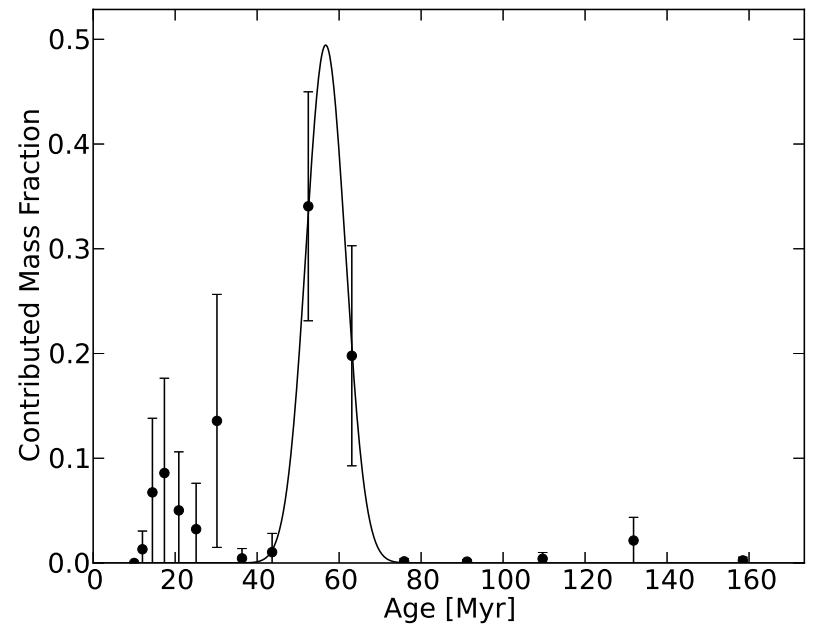

Fig. 20. Results for the fitting of the SFH of NGC 1847. The dots represent the results at individual ages. The solid line shows the best Gaussian fits to the points with a peak 56.7 Myr and a standard deviation of 4.8 Myr. The irregular star formation rate between ages of 14 and $30 \mathrm{Myr}$ is due to the MS stars that are brighter than would be expected for a $57 \mathrm{Myr}$ old population.

to the observations. If this discrepancy is due to an age spread, we can limit it to $\pm 8 \mathrm{Myr}$ as on the one hand there is no sign of recent star formation younger than $12 \mathrm{Myr}$ and on the other hand the CMD positions of the post-MS stars older than $30 \mathrm{Myr}$ are inconsistent with the observations. As we already mentioned in Sect. 4.3, this stars in the Blue-Hertzsprung-Gap can also be crowded stars, fast rotating stars, interacting binaries or merged stars.

We used for the fitting of the SFH of NGC 2004 the Parsec 1.1 isochrones. The result is shown in Fig. 26. As expected, the peak of the star formation is at around $20 \mathrm{Myr}$, with a dispersion of 1.4 Myr. Besides the prominent peak there are also points with a non-zero star formation at higher ages. However, we can rule out at a $2 \sigma$ level star formation at these ages mainly because of two reasons. First, a similar behavior is seen in the

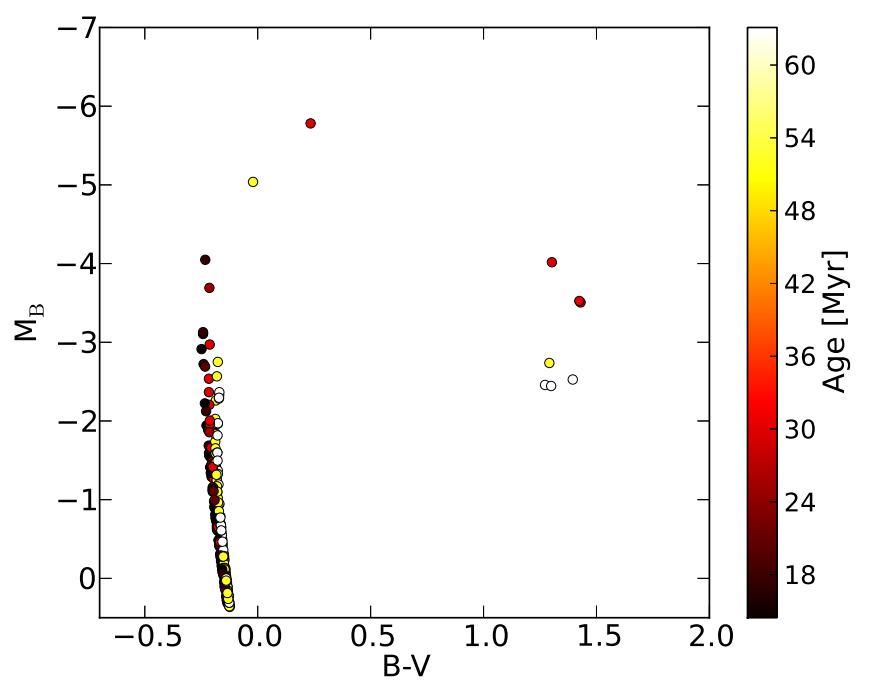

Fig. 21. CMD of an artificial cluster created using the fitted SFH of NGC 1847 normalized to the number of stars in NGC 1847 that have a $V$ band magnitude brighter than 0.5 mag. All the stars are color-coded by their age.

tests with artificial coeval clusters (see Sect. 3) which is indicative that the non-zero values at higher ages are introduced by the fitting itself. Second, a forming cluster that undergoes an extended period of low star formation followed by a burst of high star formation is a un-physical scenario (e.g. Cabrera-Ziri et al. 2014). The usage of Marigo et al. (2008) isochrones does not change the overall result.

\subsection{NGC 2100}

According to previous studies, NGC 2100 is the youngest cluster in our sample of YMCs (see Table 1). By superimposing model isochrones over the cluster CMD we find an extinction of $E(B-V)=0.24$ for this cluster, which agrees with the value found by Keller et al. (2000). Moreover, we determined a metallicity of 0.007 and a distance modulus of 18.5 mag. Figure 27 

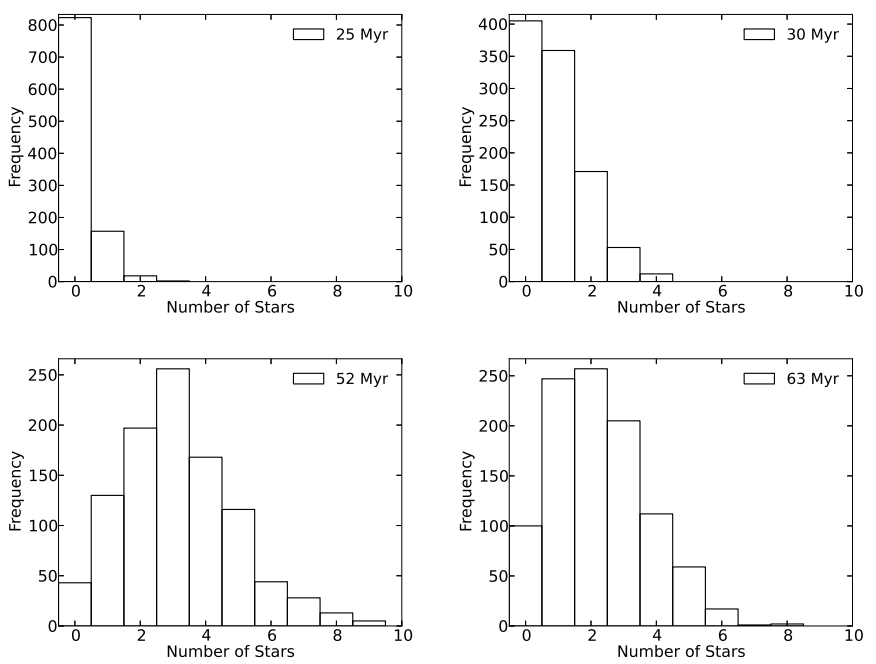

Fig. 22. Occurrence rate of post-MS stars in a simulation of 1000 artificial clusters constructed from the fitted SFH of NGC 1847. The four panels show four different ages (25 Myr, $30 \mathrm{Myr}, 52 \mathrm{Myr}$ and $63 \mathrm{Myr}$, going from top left to bottom right).

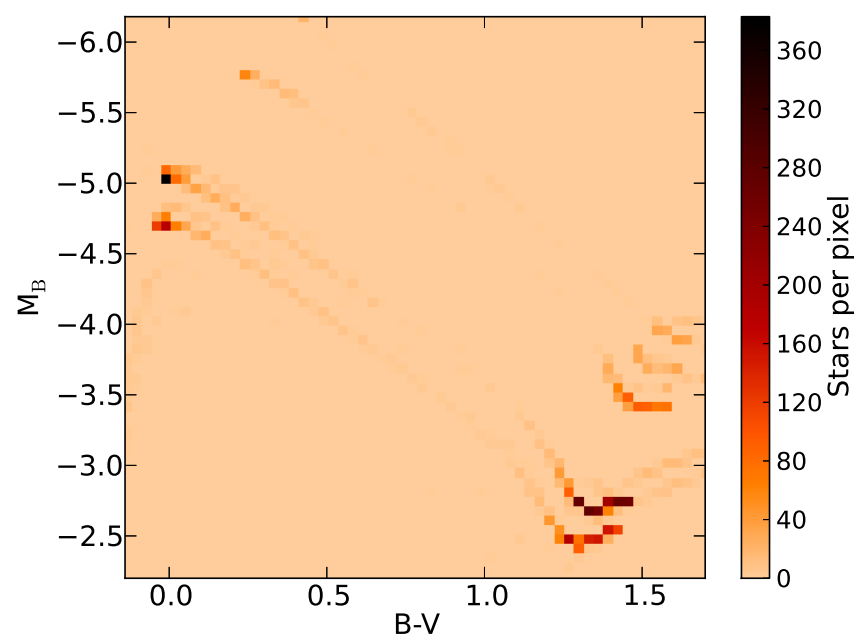

Fig. 23. Stellar density plot of the post-MS tracks of all 1000 synthetic clusters with the fitted SFH of NGC 1847 stacked together. The density is a measure of the probability to find a star in the respective part of the CMD.

shows the CMD of the cluster. The MS is located at a $B-V$ color of $\sim-0.1$ and extends up to a magnitude of $\sim 14$ (in apparent $V$ magnitude). Besides the MS there are three bright and red stars at $V \sim 13$ and $B-V \sim 1.7$ which we believe are associated with the cluster. The other fainter red stars are most likely field stars that were not removed completely. NGC 2100 shows a broad MS with several stars scattered to redder colors. This feature could have been caused by different things: 1) field stars; 2) signs of a possible age spread inside the cluster population or 3) differential reddening across the cluster.

To investigate which effect causes this broadening we divided the MS in to three parts, one at the blue, one at the red side, and the region in between the two, with the same magnitude cuts well above the completeness limit (see Fig. 27 the circles (in blue), squares (in red) and the asterisks (in green)) and plotted their spatial positions (see Fig. 28). If the red stars were stars not associated with the cluster we would expect them to be distributed evenly over the selected region. They should also appear in the CMD of the region that we used to subtract the field population from the cluster population. However, there is no indication for them in the field CMD (see Fig. 4). On the other hand if the stars would belong to a somewhat older population they should be distributed in a similar manner as the blue stars, i.e. less concentrated towards the center. But what we actually see is that the red stars preferentially populate the central region of the cluster. The blue stars avoid the central and south-west parts of the cluster and are mostly found in the north-west. The green stars that populate the intermediate part of the MS are centrally condensed and follow the overall distribution of the cluster stars. This all is a strong indication that the stars in NGC 2100 are affected by differential reddening with higher extinction values towards the central parts of the cluster and lower extinction towards the north-east. The fact that the extinction is not constant across the cluster will introduce an additional scatter in the SFH of NGC 2100. In our analysis we have to consider this effect.

Therefore, before we fit the SFH of the cluster, we take an extra step here and correct the CMD of NGC 2100 for differential extinction. We adopt the method from Milone et al. (2012), which is explained in great detail in that paper. Here we give a short description of the basic steps that we have performed: First, we define the reddening vector in the CMD of NGC 2100 and rotate the CMD around an arbitrarily chosen point in the CMD such that the reddening vector is horizontal in the new "color"-"magnitude" diagram (see Fig. 29). We will refer to the new axes as "color" and "magnitude". In the second step we define a fiducial line along the MS in the rotated CMD by calculating the median "color" of the stars in 0.25 "magnitude" bins and quadratically interpolating between them. Then we choose all stars in the real magnitude interval of $15.5 \lesssim B$ [mag] $\lesssim 18.5$ as reference stars that are used to determine the extinction differences across NGC 2100. For each reference star we identified the closest spatial neighbors and calculated the median "color" distance to the fiducial line of these neighbors (not including the respective star itself). Depending on where in the cluster the star is located (center or outskirts) we used 15 or 20 closest neighbors. As the extinction vector is parallel to the "color" axis in the rotated CMD the median displacement of a star's neighbors is equivalent to a shift caused by extinction. We then divided the cluster field in annulus segments in which the extinction has approximately the same level and calculated the median reddening in each segment. As a last step we corrected all stars in the cluster CMD for this median extinction, depending on its position in the cluster. We performed the above procedure two times in a sequence. A third correction would not have changed the final result by much. As we only have a small number of stars ( 400) in this cluster that we used for the determination of extinction variations we can only correct on large scales and might miss possible variations on smaller scales. Therefore the corrected CMD might still be affected by differential extinction to a certain degree. Figure 30 shows the CMD of NGC 2100 after it has been corrected for differential extinction. The MS is now better defined, especially at intermediate magnitudes, whereas the upper part still is broadened. We also note that, in order to match theoretical models, we have to adjust the mean extinction from $E(B-V)=0.24$ to 0.17 .

Figure 31 displays the results of the SFH fit of NGC 2100 together with a Gaussian that matches best the highest peak. The star formation shows two peaks, a larger one with a maximum at about $20 \mathrm{Myr}$ followed by a smaller peak at about 60 Myr. Additionally there are some non-zero values between 90 and 160 Myr. To quantify the reliability of the fitting we compare the results with theoretical Hess diagrams. These diagrams at three different ages, together with the corrected CMD 

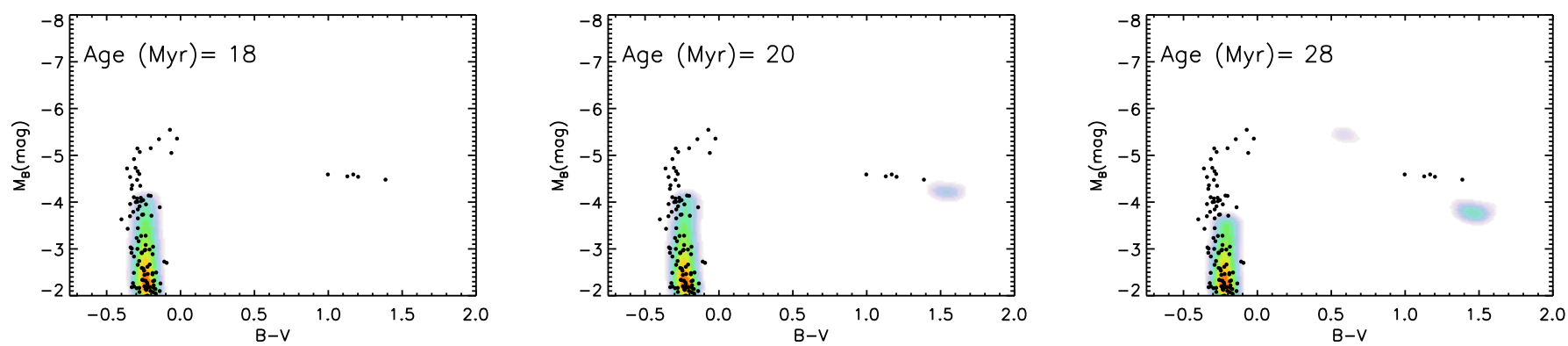

Fig. 24. CMD of NGC 2004 together with theoretical isochrones at three ages from 18 to 28 Myr.
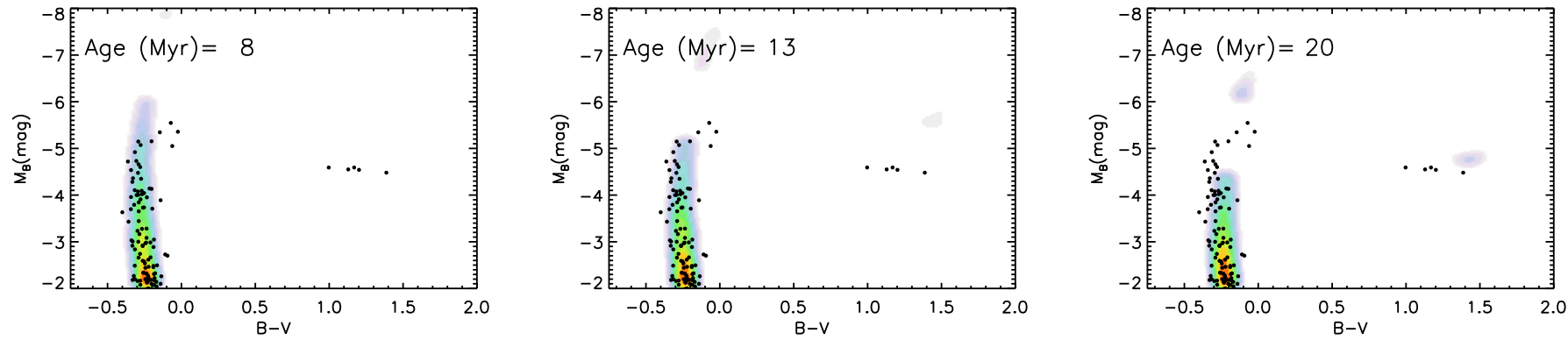

Fig. 25. Same as Fig. 24 but using Marigo et al. (2008) isochrones from 8 to 20 Myr.

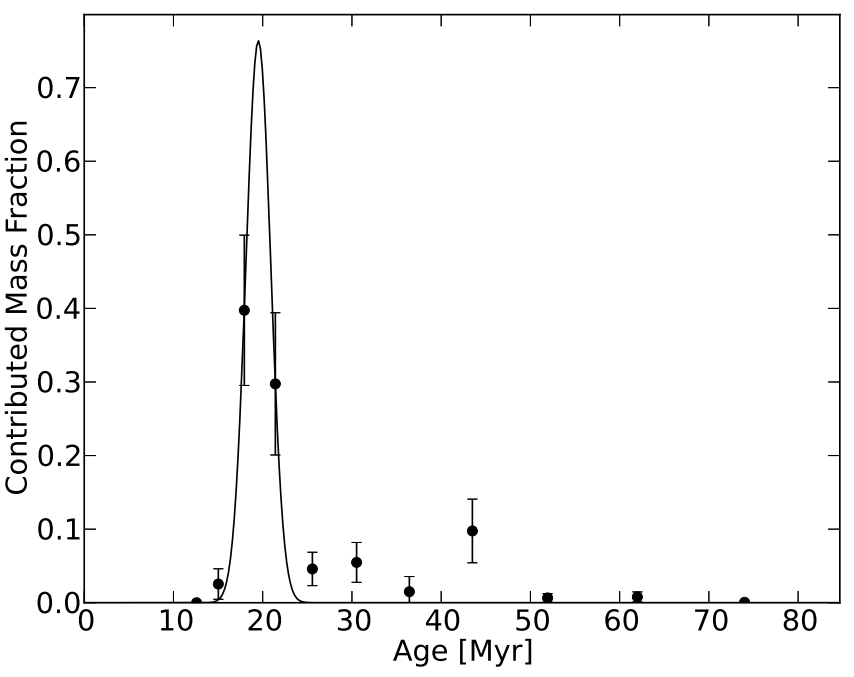

Fig. 26. Results for the fitting of the SFH of NGC 2004. The dots represent the results at individual ages and the solid line shows the best Gaussian fit to the points with a peak at $19.5 \mathrm{Myr}$ and a standard deviation of $1.4 \mathrm{Myr}$.

of NGC 2100, are shown in Fig. 32. We see that at an age of $20 \mathrm{Myr}$, the Hess diagram matches best the position of the evolved red stars and the top of the MS. At a younger age of about 16 Myr (upper left) the three blue loop stars cannot be explained as there are no stars that evolved off from the MS to redder colors. This is also caused by the usage of the Parsec 1.1 isochrone set that contains only stars up to $12 M_{\odot}$. As in the previous clusters there are a few bright stars above the MS that cannot be explained by the models.

The fitting of the SFH of NGC 2100 shows, in addition to the highest peak at $20 \mathrm{Myr}$, a second peak at $63 \mathrm{Myr}$. The third panel of Fig. 32 shows the CMD of NGC 2100 with a Hess diagram at $63 \mathrm{Myr}$ superimposed. The fitted SFH at this age is most likely due to the three stars at $B-V \sim 0.1$ and $B \sim-5$ mag on

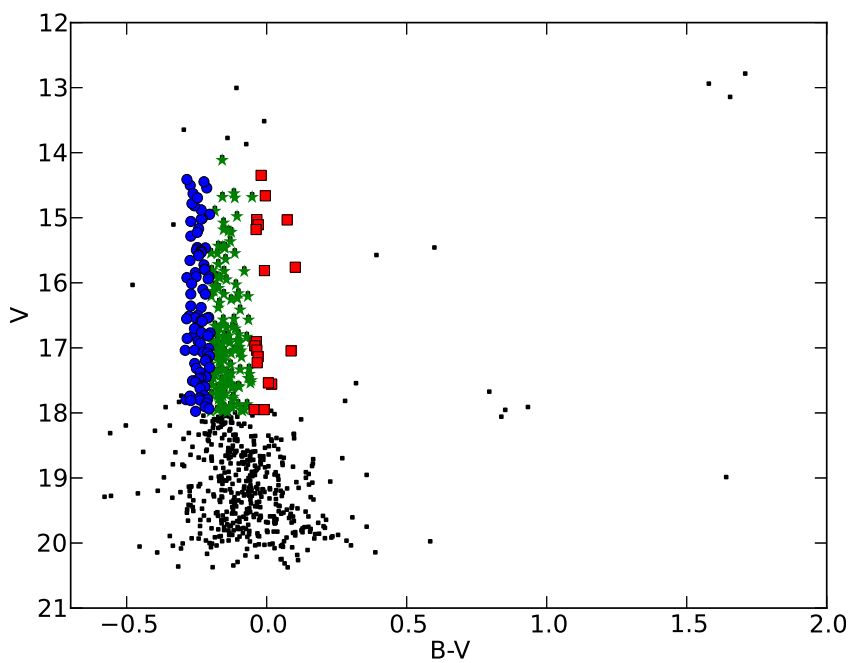

Fig. 27. CMD of the cluster NGC 2100. We used the stars that are marked by circles (in blue), the asterisk symbols (in green) and squares (in red) to determine if differential reddening could be responsible for the color spread in the main sequence.

the red side of the MS. At the same time the models predict blue loop stars to be at $B-V \sim 1.4$ and $B \sim-2.5$ mag where no stars are observed. To check if the peak at $63 \mathrm{Myr}$ is compatible with the observations from a statistical point of view, we did the same Monte Carlo test as we already did with NGC 1847 and created synthetic clusters with a SFH that is the same as predicted for NGC 2100 by the fitting. Again, the number of stars in the artificial clusters are normalized to the number of stars brighter than an absolute $V$ band magnitude of 0.0 to avoid incompleteness effects. In these simulations, we searched for blue loop stars at an age of $63 \mathrm{Myr}$ with a $B-V$ color $>0.2$ in order to not get confused with the evolved stars above the MS. Figure 33 shows the occurrence rate of post-MS stars at an age of $63 \mathrm{Myr}$ from the simulations. In $84 \%$ of the cases the test predicts at least one 


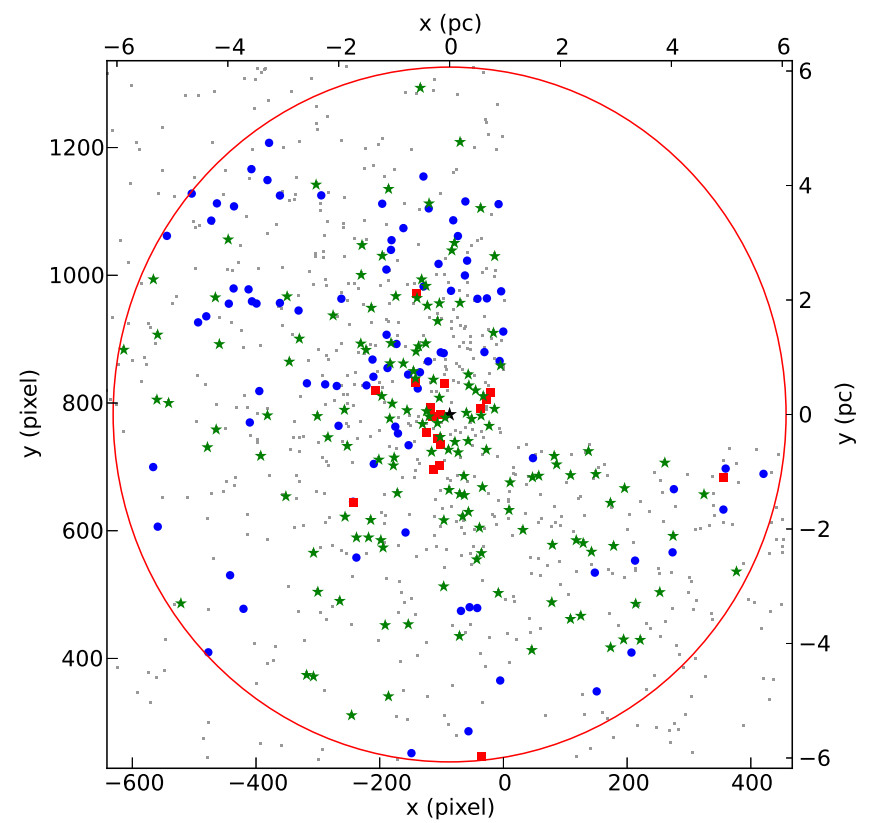

Fig. 28. Spatial positions of the red, green and blue stars marked in Fig. 27. The red stars preferentially populate the very central region of the cluster whereas the blue stars are found everywhere but the central parts. This might suggest that NGC 2100 is affected by differential reddening.

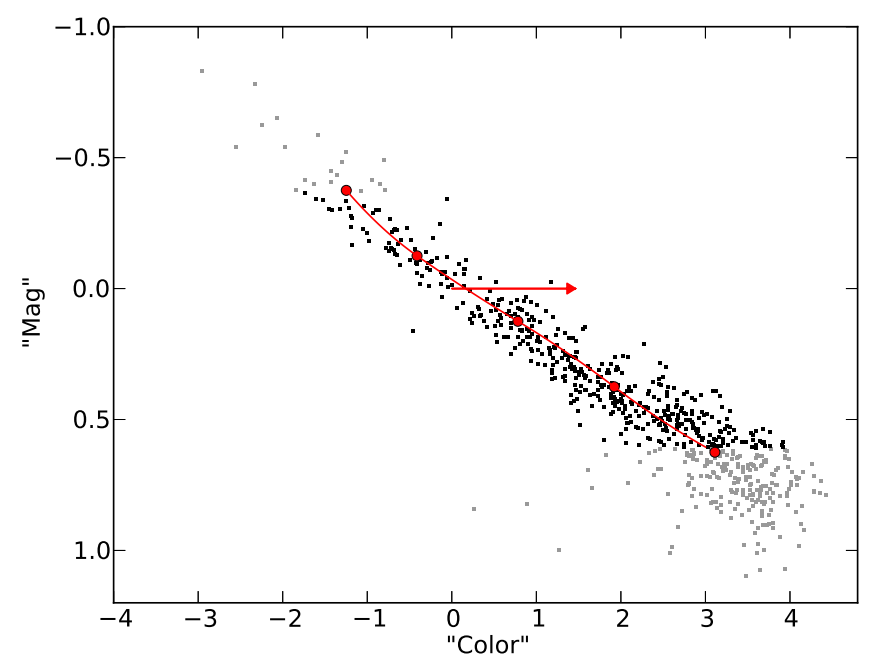

Fig. 29. CMD of NGC 2100 rotated such that the extinction vector (red arrow) is horizontal. The line (in red) along the MS is the fiducial line as it is determined in the text. The stars that are used to analyze the extinction variations across the cluster are colored black.

post-MS star. Therefore we can almost certainly rule out this age.

Beyond the two peaks, the fitting of the SFH also yields a very low level of star formation at ages between 90 and $160 \mathrm{Myr}$. But this result, as discussed in Sect. 4.7, is most likely not real.

\section{Discussion and conclusions}

\subsection{Discussion of the results}

A common feature in the CMDs of intermediate age (1-2 Gyr) LMC clusters is an extended or bifurcated MSTO which could be interpreted as an age spread of the order 100-500 Myr (e.g. Goudfrooij et al. 2011a,b). In this study we test this hypothesis.

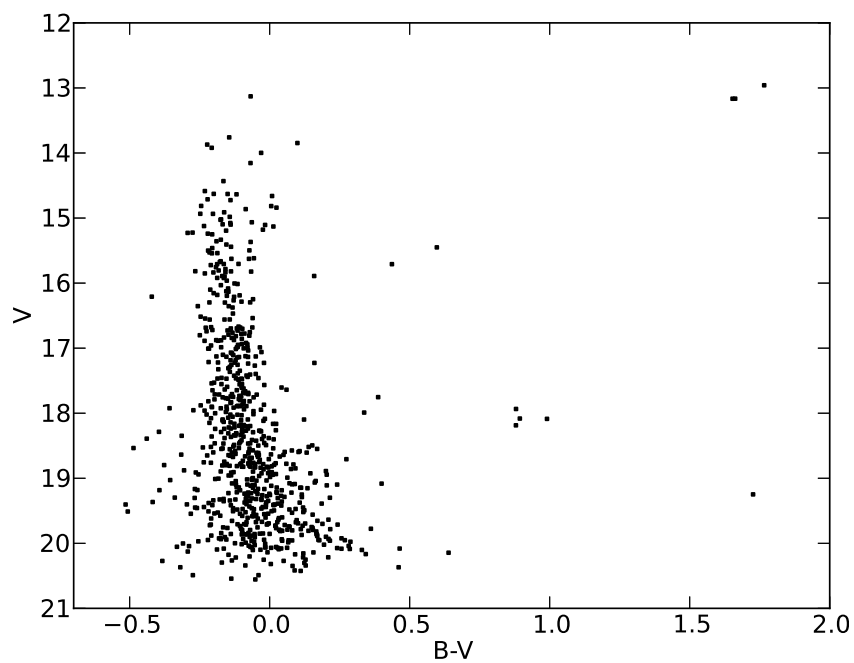

Fig. 30. CMD of NGC 2100 after correcting it for differential extinction.

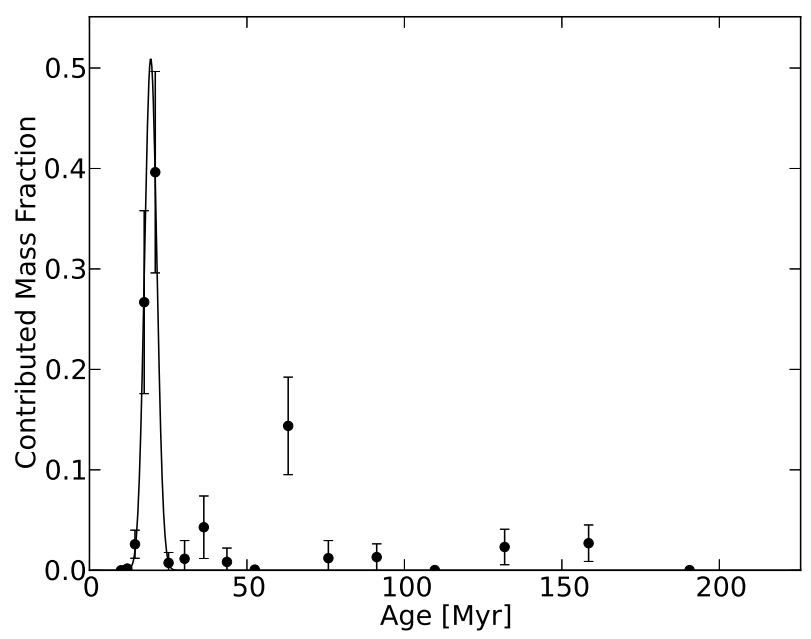

Fig. 31. Results of the SFH fit of NGC 2100. The dots represent the results at individual ages and the solid line shows the best Gaussian fit to the points with a peak at $19.5 \mathrm{Myr}$ and a standard deviation of 2.0 Myr.

An extended history of star formation in intermediate age clusters implies that an age spread of the same order should be present in young $(<1 \mathrm{Gyr})$ clusters with similar properties as well. We analyzed a sample of eight young massive LMC clusters in continuation of the study by Bastian \& Silva-Villa (2013) and searched for potential spreads in ages inside them. We compared the CMDs of these clusters with model Hess diagrams and fitted the SFH of all clusters using the FITSFH code. The results of the fitting of the highest peak are summarized in Table 3. Most of the clusters in our sample have a single and well defined peak in the fitted SFH. But there are also some clusters which show periods of low extended star formation, like NGC 1850, or a second burst, like NGC 1847 or NGC 2100 , in addition to the main star formation peak. Therefore we performed Monte Carlo simulations of artificial clusters with the same fitted star formation as the real ones to assess the reliability of the SFH fit. For NGC 1850 we were able to rule out the fitted star formation at a few 100 Myr. However, using this statistical tool, we cannot make any final statement if the younger star formation in NGC 1847 is real or not, although there are physical effects (rotation, blending, binaries) that would cause the MS extend to brighter magnitudes than would be expected from a nominal isochrone at this age. Bastian \& Strader (2014) studied the gas 

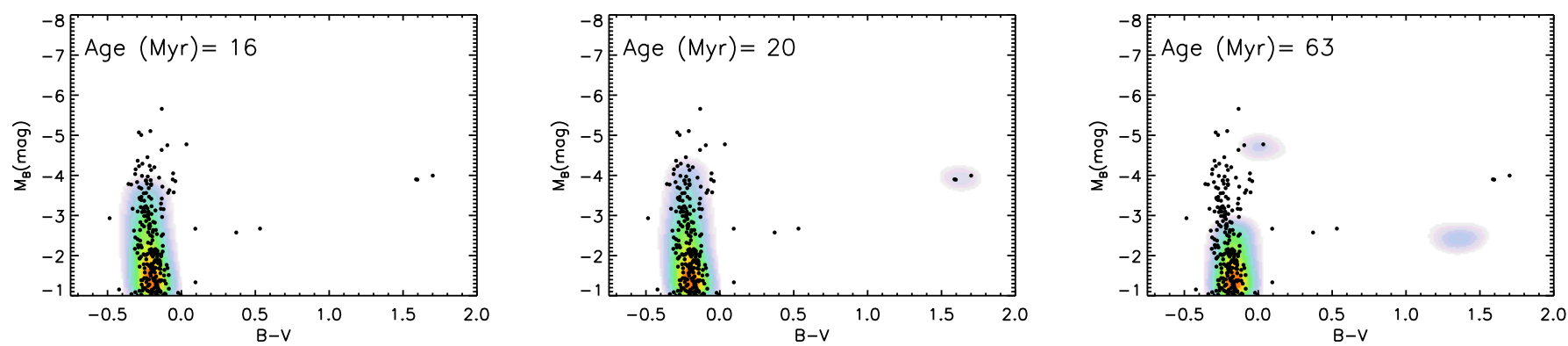

Fig. 32. CMD of NGC 2100 after correction for differential extinction. Overplotted are model Hess diagrams at three different ages in the range from 16 to $63 \mathrm{Myr}$.

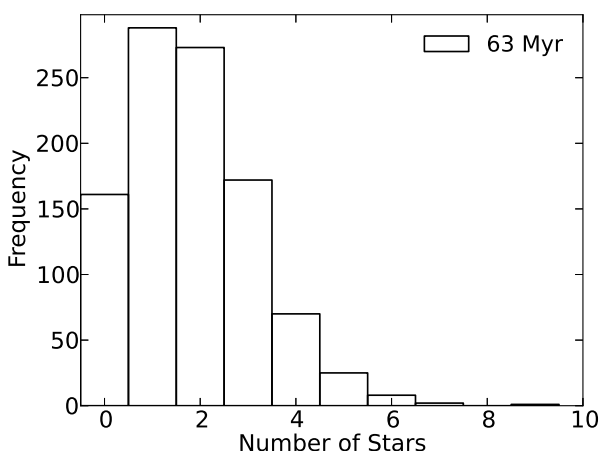

Fig. 33. Occurrence rate of post-MS stars at an age of $63 \mathrm{Myr}$ in a simulation of 1000 artificial clusters constructed from the fitted SFH of NGC 2100.

Table 3. Summary of the results from the SFH fit to the highest peak.

\begin{tabular}{lcc}
\hline \hline Cluster & $\begin{array}{c}\text { Fitted SFH } \\
\text { of highest peak }\end{array}$ & $\begin{array}{c}\text { Fitted SFH of the corresponding } \\
\text { highest peak in artificial cluster }\end{array}$ \\
\hline NGC 1831 & $924 \pm 126 \mathrm{Myr}$ & $949 \pm 57 \mathrm{Myr}$ \\
NGC 1847 & $56.7 \pm 4.8 \mathrm{Myr}$ & $60.0 \pm 1.0 \mathrm{Myr}$ \\
NGC 1850 & $93.4 \pm 18.3 \mathrm{Myr}$ & $96.2 \pm 5.7 \mathrm{Myr}$ \\
NGC 2004 & $19.5 \pm 1.4 \mathrm{Myr}$ & $20.1 \pm 1.5 \mathrm{Myr}$ \\
NGC 2100 & $19.5 \pm 2.0 \mathrm{Myr}$ & $20.6 \pm 1.6 \mathrm{Myr}$ \\
NGC 2136 & $123.3 \pm 22.6 \mathrm{Myr}$ & $130.2 \pm 9.6 \mathrm{Myr}$ \\
NGC 2157 & $98.3 \pm 13.2 \mathrm{Myr}$ & $102.2 \pm 8.4 \mathrm{Myr}$ \\
NGC 2249 & $1110 \pm 139 \mathrm{Myr}$ & $1235 \pm 111 \mathrm{Myr}$ \\
\hline NGC 1856 & $281 \pm 33.6 \mathrm{Myr}$ & - \\
NGC 1866 & $177 \pm 18.4 \mathrm{Myr}$ & - \\
\hline
\end{tabular}

Notes. The last two clusters (NGC 1856 and NGC 1866) are not studied in this work. They were already analyzed by Bastian \& Silva-Villa (2013).

and dust content in a sample of 13 LMC and SMC clusters, including NGC 1847 , with masses greater than $10^{4} M_{\odot}$ and ages between 30 and $300 \mathrm{Myr}$. They found no evidence for any gas or dust left inside the clusters, even in the most youngest ones. They conclude that clusters are very efficient in removing their gas content right after their formation. This result suggests that the younger period of star formation might not be real. However, the overall age spread, also including the younger burst, is smaller than $\sim 45 \mathrm{Myr}$ (if it is real) which is inconsistent with the proposed age spread of 100-500 Myr for intermediate age clusters.

NGC 2100 is a special case in our sample of young clusters as its CMD is affected by differential extinction across the cluster field. We corrected the photometry for the varying reddening using the method described in Milone et al. (2012). We were only able to account for large scale variations in our correction because of the limited number of stars available. Therefore, there might be some differences in extinction on smaller scales left that introduces some scatter in the final results. The SFH fit of this cluster yields two peaks, one larger at $20 \mathrm{Myr}$ and a smaller one at 63 Myr. Monte Carlo simulations of artificial clusters showed that the second peak is most likely not real.

We also note that the MS of some of the clusters extends to brighter magnitudes than would be expected from the position of the post-MS stars. In the case of NGC 1847, as already discussed, the bright stars introduce an additional younger peak in the fitted SFH. As we used Parsec 1.1 isochrones that cover only stars with masses smaller than $12 M_{\odot}$ which corresponds to the turn-off mass at $\sim 16 \mathrm{Myr}$, we additionally compared the CMD of NGC 2004 with the isochrone set from Marigo et al. (2008) that includes stellar masses up to $100 M_{\odot}$. We found that an age of $12.5 \mathrm{Myr}$ is required to explain the brightest MS stars. At this point, we cannot entirely rule out that the discrepancy between the MSTO and the evolved stars is due to an age spread. However, we can restrict the potential age spread to be $\lesssim 8 \mathrm{Myr}$ as we see no sign of recent star formation, nor for ages older than $\sim 28 \mathrm{Myr}$.

By fitting the SFHs to the LMC clusters in our sample we get age spreads (given by the dispersion of the best-fitting Gaussians to the highest star formation peak) that are all upper limits of the real age spreads that might be present in the clusters. We find that the age dispersion that we got for the clusters is increasing with increasing age of the corresponding age of the cluster. The youngest clusters in our sample have age spreads smaller than a few Myr, whereas the two oldest (NGC 1831 and NGC 2249) have a fitted dispersion in age of the order of 120-140 Myr. The lower four panels of Fig. 5 show the results of the SFH fits of four simulated clusters. We see the same trend of increasing "age spread" in the results for the simulated coeval clusters. A comparison with the results of our cluster sample shows that, on the one hand, the age dispersion is comparable for the youngest age. On the other hand, the older clusters show spreads in age that are larger ( 2 to 4 times) than we would expect from our simulations. This spread therefore must have resulted from other effects. The apparent larger age range might be introduced by an underestimation of the photometric errors, interacting binaries in the cluster and/or rotating stars as already proposed by e.g. Larsen et al. (2011).

We determined the ages of NGC 1831 and NGC 2249 to be $\sim 900 \mathrm{Myr}$ and $\sim 1.1 \mathrm{Gyr}$, respectively. Therefore they can already be classified as intermediate age LMC clusters. Moreover, the age spread larger than $100 \mathrm{Myr}$ that we found for both clusters would support the interpretation of the extended MSTO by Goudfrooij et al. (2011a,b). However, this is in conflict with the results we got for the younger clusters in our sample and in the sample of Bastian \& Silva-Villa (2013). Their age dispersion is much smaller as would be expected from the proposed age 
spreads in the intermediate age LMC clusters. In addition, as already mentioned, all age spreads are upper limits and there are non-negligible extrinsic (photometric errors, differential extinction) and intrinsic (interacting binaries, rotating stars) contributions that increase the apparent spread. Therefore the fitted extended SFH of the two intermediate age clusters in our sample does not support the Goudfrooij et al. (2011a,b) scenario.

Goudfrooij et al. (2011b) suggest that clusters that have an escape velocity greater than a critical value of $10 \mathrm{~km} \mathrm{~s}^{-1}$ at the beginning of their lifetimes should be capable to host multiple generations of stars. Table 1 lists the escape velocities of all clusters in our data set plus the clusters from Bastian \& Silva-Villa (2013). We estimate the value of $v_{\text {esc }}$ the clusters had when they were 10 Myr old using the method presented in Goudfrooij et al. (2011b) that accounts for mass loss. We do not assume any evolution in radius. Whereas the value of the younger clusters does not change by much, the escape velocity of the older clusters ( $>100 \mathrm{Myr}$ ) will be larger by a factor between 1.26 and 1.4. After applying this correction to the clusters, NGC 1850, NGC 1856 and NGC 1866 have escape velocities that are larger than $10 \mathrm{~km} \mathrm{~s}^{-1}$. None of them shows signs of an extended history of star formation disagreeing with the predictions made by Goudfrooij et al. (2011b). Another criterion for an extended SFH is given by Conroy \& Spergel (2011) who stated that clusters more massive than $10^{4} M_{\odot}$ should have age spreads. However, all our sample clusters are well above this threshold.

Analyzing the CMDs of young and massive LMC clusters we found a new age for the main cluster of the binary system NGC 1850. Our result of about $100 \mathrm{Myr}$ is significantly higher than the commonly used value of $\sim 30-50$ Myr. This increase in age also affects the mass of the cluster which increases by a factor of two, from $7.2 \times 10^{4} M_{\odot}$ to $1.4 \times 10^{5} M_{\odot}$. This new mass is in agreement with the predicted mass-to-light ratio for a 100 Myr population.

\subsection{Comparison with the intermediate age clusters}

In Table 1 we show the estimated current masses and escape velocities of the clusters in our sample. Comparison with Table 2 of Goudfrooij et al. (2011b) shows the clusters studied here have masses and escape velocities in the same range as the intermediate age clusters that display the extended MSTO phenomenon. However, Goudfrooij et al. (2011b) have suggested that large "correction factors" need to be applied to the intermediate age clusters in order to estimate their initial masses and escape velocities. The scenario advocated by these authors is that a large cluster was formed in a near-instantaneous burst, and that this cluster was able to retain the ejecta from some of the evolved stars within it, as well as accrete new gas from its surroundings, in order to form a second generation of stars (which lasts for 300-600 Myr). During the formation of the second generation, the majority ( $>80-90 \%)$ of the first generation stars are lost (i.e. they are no longer in the present day cluster). For this, the initial massive cluster is assumed to be 1) tidally limited;2) in a strong tidal field (suitable for the inner regions of the Milky Way, not tailored to that expected in the LMC/SMC) and 3) mass segregated (which accelerates mass loss by causing a cluster to expand over its Jacobi radius). The initial cluster then loses the vast majority of its initial population of stars, and what is left today is only the second generation stars. It is unclear in this model why the second generation of stars would show a Gaussian type age distribution as observed. When applying these correction factors, Goudfrooij et al. (2011b) suggest that a cluster must have an escape velocity in excess of $10-15 \mathrm{~km} \mathrm{~s}^{-1}$ in order to form a second generation of stars.

However, given the relatively weak tidal fields in the LMC/SMC, and the fact the intermediate age (and older) clusters do not appear to be tidally limited (e.g., Glatt et al. 2009), it is more likely that the intermediate age clusters have not undergone strong mass loss, hence their current masses and escape velocities are similar to their initial values. In this interpretation, we see that there is no relation between the mass or escape velocity of clusters that do or do not display the extended MSTO feature (i.e. potential evidence for an age spread).

Hence, we conclude the scenario put forward by Goudfrooij et al. (2011b), that a minimum escape velocity is required for clusters to undergo extended secondary star formation events, is highly dependent on the adopted "correction factors". If standard "correction factors" are adopted for the LMC/SMC, there is no relation between the escape velocity and the existence of age spreads or the extended MSTO feature.

\subsection{Comparison with other YMCs in the LMC}

Our findings are in line with previous studies of young massive LMC clusters. Milone et al. (2013) ruled out a potential age spread present in NGC 1844 that shows broadened MS features. They derived an age of NGC 1844 of $\sim 150 \mathrm{Myr}$. Li et al. (2013) proposed that NGC $1805\left(44.7 \mathrm{Myr}, 4.7 \times 10^{3} M_{\odot}\right)$ and NGC $1818\left(17.8 \mathrm{Myr}, 2.6 \times 10^{4} M_{\odot}\right)$ are simple stellar populations with no evident age spreads. These results are all in disagreement with the findings e.g. by Rubele et al. (2013) and Goudfrooij et al. (2011a,b) who explained the extended MSTO feature observed in many intermediate age LMC clusters with an age spread of 100-500 Myr inside the cluster. As a solution to this discrepancy we propose that rotating stars are (at least partially) responsible for the anomalous features observed in stellar clusters as it is already suggested by e.g. Bastian \& de Mink (2009) and Yang et al. (2013). We will test this hypothesis in an upcoming paper (Niederhofer et al., in prep.) where we will analyze observed CMDs of YMCs using the new population synthesis code (SYCLIST) of the Geneva group (Georgy et al. 2014) that includes all effects of stellar rotation.

Acknowledgements. This research was supported by the DFG cluster of excellence "Origin and Structure of the Universe". We thank the anonymous referee for useful comments that helped to improve the manuscript.

\section{References}

Bastian, N., \& de Mink, S. E. 2009, MNRAS, 398, L11 Bastian, N., \& Silva-Villa, E. 2013, MNRAS, 431, 122 Bastian, N., \& Strader, J. 2014, MNRAS, 443, 3594

Bastian, N., Lamers, H. J. G. L. M., de Mink, S. E., et al. 2013a, MNRAS, 436, 2398

Bastian, N., Cabrera-Ziri, I., Davies, B., \& Larsen, S. S. 2013b, MNRAS, 436, 2852

Baumgardt, H., Parmentier, G., Anders, P., \& Grebel, E. K. 2013, MNRAS, 430, 676

Bressan, A., Marigo, P., Girardi, L., et al. 2012, MNRAS, 427, 127

Brocato, E., Di Carlo, E., \& Menna, G. 2001, A\&A, 374, 523

Cabrera-Ziri, I., Bastian, N., Davies, B., et al. 2014, MNRAS, 441, 2754

Conroy, C. 2012, ApJ, 758, 21

Conroy, C., \& Spergel, D. N. 2011, ApJ, 726, 36

Correnti, M., Goudfrooij, P., Kalirai, J. S., et al. 2014, ApJ, 793, 121

D'Ercole, A., Vesperini, E., D’Antona, F., McMillan, S. L. W., \& Recchi, S. 2008, MNRAS, 391, 825

Decressin, T., Charbonnel, C., Siess, L., et al. 2009, A\&A, 505, 727

de Grijs, R., Wicker, J. E., \& Bono, G. 2014, AJ, 147, 122

de Mink, S. E., Pols, O. R., Langer, N., \& Izzard, R. G. 2009, A\&A, 507, L1

Dirsch, B., Richtler, T., Gieren, W. P., \& Hilker, M. 2000, A\&A, 360, 133 
Elson, R. A., \& Fall, S. M. 1988, AJ, 96, 1383

Elson, R. A. W. 1991, ApJS, 76, 185

Fischer, P., Welch, D. L., \& Mateo, M. 1993, AJ, 105, 938

Fischer, P., Pryor, C., Murray, S., Mateo, M., \& Richtler, T. 1998, AJ, 115, 592

Georgy, C., Granada, A., Ekström, S., et al. 2014, A\&A, 566, A21

Girardi, L., Bertelli, G., Bressan, A., et al. 2002, A\&A, 391, 195

Glatt, K., Grebel, E. K., Gallagher, J. S., III, et al. 2009, AJ, 138, 1403

Goudfrooij, P., Puzia, T. H., Kozhurina-Platais, V., \& Chandar, R. 2009, AJ, 137, 4988

Goudfrooij, P., Puzia, T. H., Kozhurina-Platais, V., \& Chandar, R. 2011a, ApJ, 737,3

Goudfrooij, P., Puzia, T. H., Chandar, R., \& Kozhurina-Platais, V. 2011b, ApJ, 737, 4

Gratton, R. G., Carretta, E., \& Bragaglia, A. 2012, A\&ARv, 20, 50

Jasniewicz, G., \& Thevenin, F. 1994, A\&A, 282, 717

Keller, S. C., Bessell, M. S., \& Da Costa, G. S. 2000, AJ, 119, 1748

Keller, S. C., Mackey, A. D., \& Da Costa, G. S. 2011, ApJ, 731, 22

Kerber, L. O., Santiago, B. X., \& Brocato, E. 2007, A\&A, 462, 139

King, I. R. 1966, AJ, 71, 64

Larsen, S. S., de Mink, S. E., Eldridge, J. J., et al. 2011, A\&A, 532, A147

Li, C., de Grijs, R., \& Deng, L. 2013, MNRAS, 436, 1497

Li, C., de Grijs, R., \& Deng, L. 2014, ApJ, 784, 157

Mackey, A. D., \& Broby Nielsen, P. 2007, MNRAS, 379, 151
Mackey, A. D., \& Gilmore, G. F. 2003, MNRAS, 338, 85

Mackey, A. D., Broby Nielsen, P., Ferguson, A. M. N., \& Richardson, J. C. 2008, ApJ, 681, L17

Marigo, P., Girardi, L., Bressan, A., et al. 2008, A\&A, 482, 883

McLaughlin, D. E., \& van der Marel, R. P. 2005, ApJS, 161, 304

Meynet, G., \& Maeder, A. 1997, A\&A, 321, 465

Milone, A. P., Bedin, L. R., Piotto, G., \& Anderson, J. 2009, A\&A, 497, 755

Milone, A. P., Piotto, G., Bedin, L. R., et al. 2012, A\&A, 540, A16

Milone, A. P., Bedin, L. R., Cassisi, S., et al. 2013, A\&A, 555, A143

Mucciarelli, A., Carretta, E., Origlia, L., \& Ferraro, F. R. 2008, AJ, 136, 375

Mucciarelli, A., Cristallo, S., Brocato, E., et al. 2011, MNRAS, 413, 837

Mucciarelli, A., Dalessandro, E., Ferraro, F. R., Origlia, L., \& Lanzoni, B. 2014, ApJ, 793, L6

Nelson, M., \& Hodge, P. 1983, PASP, 95, 5

Piotto, G., Milone, A. P., Anderson, J., et al. 2012, ApJ, 760, 39

Platais, I., Melo, C., Quinn, S. N., et al. 2012, ApJ, 751, L8

Rubele, S., Girardi, L., Kozhurina-Platais, V., et al. 2013, MNRAS, 430, 2774

Salpeter, E. E. 1955, ApJ, 121, 161

Sebo, K. M., \& Wood, P. R. 1995, ApJ, 449, 164

Silva-Villa, E., \& Larsen, S. S. 2010, A\&A, 516, A10

Vallenari, A., Aparicio, A., Fagotto, F., \& Chiosi, C. 1994, A\&A, 284, 424

Yang, W., Meng, X., Bi, S., et al. 2011, ApJ, 731, L37

Yang, W., Bi, S., Meng, X., \& Liu, Z. 2013, ApJ, 776, 112 